\title{
Evolution equations in ostensible metric spaces: First-order evolutions of nonsmooth sets with nonlocal terms.
}

\author{
Thomas Lorenz $^{1}$
}

\begin{abstract}
Similarly to quasidifferential equations of Panasyuk, the so-called mutational equations of Aubin provide a generalization of ordinary differential equations to locally compact metric spaces. Here we present their extension to a nonempty set with a possibly nonsymmetric distance. In spite of lacking any linear structures, a distribution-like approach leads to so-called right-hand forward solutions.

These extensions are mainly motivated by compact subsets of the Euclidean space whose evolution is determined by the nonlocal properties of both the current set and the normal cones at its topological boundary. Indeed, simple deformations such as isotropic expansions exemplify that topological boundaries need not evolve continuously in time and thus, Aubin's original concept cannot be applied directly. Here neither regularity assumptions about the boundaries nor the inclusion principle are required. The regularity of compact reachable sets of differential inclusions is studied extensively instead.

This example of nonlocal set evolutions in the Euclidean space serves as an introductory motivation for extending ordinary differential equations (and evolution equations) beyond the traditional border of vector spaces - and for combining it with other examples in systems.
\end{abstract}

Keywords Mutational equations, quasidifferential equations, funnel equations, nonlocal geometric evolutions, reachable sets of differential inclusions, sets of positive erosion, sets of positive reach MSC Subjects $\quad 34 \mathrm{~A} 60,34 \mathrm{G} 25,47 \mathrm{D} 06,49 \mathrm{~J} 53,34 \mathrm{G} 20$

\section{Contents}

1 Introduction

2 Ordinary differential equations in metric spaces:

A summary of Aubin's mutational equations

3 Generalizing mutational equations : Nonsymmetric distance and distribution-like solutions.

3.1 Obstacles to first-order geometric evolutions due to boundaries . . . . . . . . . . . . 9

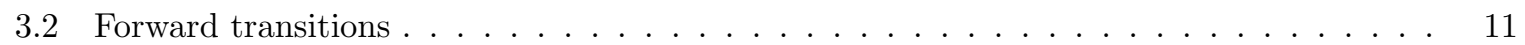

3.3 Right-hand forward solutions . . . . . . . . . . . . . . . . . . . . 13

4 Nonlocal evolution of compact subsets of $\mathbb{R}^{N} \quad 18$

A Reachable sets of differential inclusions in $\mathbb{R}^{N} \quad \mathbf{2 6}$

A.1 Hamiltonian system helps preserving $C^{1,1}$ boundaries shortly $\ldots \ldots \ldots \ldots \ldots$

A.2 How to guarantee reversibility of $C^{1,1}$ sets in time (shortly) $\ldots \ldots \ldots \ldots \ldots$

A.3 How to make points evolve into sets of positive erosion . . . . . . . . . . . . 30

Bibliography

\footnotetext{
${ }^{1}$ Interdisciplinary Center for Scientific Computing (IWR)

Ruprecht-Karls-University of Heidelberg

Im Neuenheimer Feld 294, 69120 Heidelberg (Germany)

thomas.lorenz@iwr.uni-heidelberg.de

First version: May 12, 2006. Revised: November 5, 2007
} 



\section{Introduction}

Whenever different types of evolutions meet, they usually do not have an obvious vector space structure in common providing a basis for differential calculus. In particular, "shapes and images are basically sets, not even smooth" as Aubin stated [2]. So he regards this obstacle as a starting point for extending ordinary differential equations to metric spaces - the so-called mutational equations [2, 3, 4].

In fact, similar concepts have been developed independently, e.g. the quasidifferential equations of A.I. Panasyuak [45, 46, 47], quasi-flows of P.E. Kloeden et al. and the mostly ellipsoidal approaches of A.B. Kurzhanski et al. [36, 37, 38]. Here we seize Aubin's concept though and extend it significantly for the first time (since its initial presentation in 1992/93).

Considering the example of time-dependent compact sets in $\mathbb{R}^{N}$, Aubin uses reachable sets of differential inclusions for describing a first-order approximation with respect to the Pompeiu-Hausdorff distance $d$. However this approach (also called morphological equations) can hardly be applied to geometric evolutions depending on the topological boundary explicitly. Indeed, roughly speaking, "holes" of sets might disappear while evolving along differential inclusions and thus, analytically speaking, the topological boundary need not be continuous with respect to time.

In this paper, this difficulty is the essential motivation for

- extending mutational equations to a set $E \neq \emptyset$ with an ostensible metric, i.e. distance function $q: E \times E \longrightarrow[0, \infty[$ satisfying just the triangle inequality and $q(x, x)=0$ for each $x \in E$ (In particular, it need not be symmetric any longer.)

- introducing a concept of distribution-like solutions in ostensible metric spaces (although linear forms are not available beyond vector spaces)

- investigating the reachable sets of differential inclusions and their regularity at the boundary.

In other words, we focus on a new generalization of evolution equations beyond vector spaces $(\S 3)$ and verify the required preliminaries in detail for nonlocal geometric evolutions up to first order $(\S 4)$, i.e. compact subsets of $\mathbb{R}^{N}$ whose evolution depend on nonlocal properties of both the sets and their limiting normal cones at the topological boundary.

This geometric example uses compact reachable sets of differential inclusions in the Euclidean space and requires new results about their topological boundaries and their normal cones (see Appendix). In particular, we specify sufficient conditions on the differential inclusions such that every compact set with $C^{1,1}$ boundary preserves this regularity for a short time and evolves reversibly during this period.

\section{Some earlier approaches to set evolutions}

Many approaches to set evolutions are based on level sets of an auxiliary function. Being introduced in numerical context by Osher and Sethian [44], this notion has a solid analytical basis in form of so-called viscosity solutions that were first defined by Crandall and Lions [23, 24]. Applying this concept to set evolutions with prescribed normal velocity, an essential advantage is that many forms of geometric singularities can be handled quite easily (see e.g. [25, 26, Evans, Spruck 91/92], [18, Chen, Giga, Goto 91], [7, Barles, Soner, Souganidis 93], [8, Barles, Souganidis 98], [1, Ambrosio 2000]).

Recently, some results about viscosity solutions of nonlocal problems have been published by Barles and Ley [6] but they are restricting to the case that the normal velocity of all level sets preserves its sign (i.e. either permanently nonnegative or nonpositive). 
Viscosity solutions (in all their variants so far) are always based on the maximum principle. This implies two features that our approach is to overcome: Firstly, there is no general theory about viscosity solutions of systems and secondly, all set evolutions resulting from viscosity solutions of partial differential equations satisfy the so-called inclusion principle, i.e. if a compact initial set is contained in another one, then this inclusion is be preserved while the sets are evolving.

In fact, several further approaches use this principle as a geometric starting point for extending analytical tools from sets with smooth boundaries to nonsmooth subsets. An excellent geometric counterpart of the classical Perron method is De Giorgi's theory of barriers formulated in [27, De Giorgi 94] and elaborated in [11, 10, Bellettini, Novaga 97/98]. Another elegant approach to front propagation problems with nonlocal terms has been presented in [16, 15, Cardaliaguet 2000/01]. However, there is no obvious way of applying these earlier concepts to the easy example that the normal velocity at the boundary is $\frac{1}{1+\text { set diameter }}>0$.

Geometric measure theory lays the basis of further approaches for dealing with geometric singularities (see e.g. [31, Federer 69], [12, Brakke 78]). Using measures, however, implies that the information about all subsets with measure 0 is definitely lost. Coping with problems in shape optimization, Delfour and Zolésio suggest using the (classical or oriented) distance from the current set - instead of the corresponding characteristic function being either 0 or $1[28,29]$. Then the choice of the functional space, however, has a significant influence on the regularity of sets that can be handled (see e.g. [29, § 5.6.3]).

\section{Three challenges for nonlocal evolutions of compact sets in $\mathbb{R}^{N}$}

Considering the time-dependent compact subsets of $\mathbb{R}^{N}, t \longmapsto K(t)$, it is not directly evident how to define their "rate of change" (as counterpart of "velocity"). The mutational concept of Aubin lays the foundation of specifying an answer. In particular, it extends the notion of time derivative without any regularity conditions on its topological boundary $\partial K(t) \subset \mathbb{R}^{N}$.

Considering the previous approaches to set evolutions, we are now facing three further challenges motivating our generalization of Aubin's theory:

- Evolution of $K(t)$ via prescribed feedback depending on nonlocal properties "up to first order", i.e. the compact set $K(t) \subset \mathbb{R}^{N}$ and the graph of its limiting normal cones $N_{K(t)}(\cdot)$ - as a whole are taken into consideration for determining the evolution. (So no subsets of measure 0, for example, are neglected. The concept here will not cover boundary properties of second order like mean curvature, though.)

- No restricting to geometric evolutions obeying the inclusion principle.

- The analytical concept can also handle systems with several evolving sets simultaneously.

\section{Extending the traditional horizon: Evolution equations beyond vector spaces}

In fact, we regard nonlocal first-order geometric evolutions just as an example for applying generalized mutational equations and, it is not the only application, indeed. Minor modifications (concerning the number of distance functions) have already led to similar results for semilinear evolution equations in reflexive Banach spaces (see $[43, \S 4.5]$ ) and, a forthcoming paper will focus on the nonlinear transport equation for positive Radon measures on $\mathbb{R}^{N}[40]$. 
Introducing mutational equations in metric spaces, Aubin's motivation was to extend ordinary differential equations to compact subsets of the Euclidean space. This approach, however, has a much larger potential. Indeed, the main goal here is a common analytical framework for dynamic systems beyond the traditional border of vector spaces.

Whenever a component proves to fit in this framework (like the first-order geometric evolutions here), the mutational theory immediately opens the door to existence results about systems with other suitable components - no matter whether their mathematical origins are completely different. So a nonlocal geometric evolution can be combined, for example, with an ordinary differential equation and a semilinear evolution equation. This is the main advantage of mutational equations - in comparison to more popular concepts like viscosity solutions and thus, all our generalizations here are to preserve this feature. It is to lay the foundations of new future results about free boundary problems.

\section{Aubin's initial notion: Consider suitable "deformations" instead of affine-linear maps.}

The step from the Euclidean space to a metric space is based on a very simple idea: In $\mathbb{R}^{N}$, each vector $v \in \mathbb{R}^{N}$ induces a continuous map describing the deformation of $\mathbb{R}^{N}$ after finite time. For mainly historic reasons, this map is usually assumed to be affine-linear: $[0,1] \times \mathbb{R}^{N} \longrightarrow \mathbb{R}^{N},(h, x) \longmapsto x+h \cdot v$. Considering now a curve $x(\cdot):[0, T] \longrightarrow \mathbb{R}^{N}$, the popular definitions of time derivative (at time $t \in[0, T]$ ) are all based on choosing the vector $v \in \mathbb{R}^{N}$ such that the corresponding affine-linear map provides a first-order approximation of $x(t+\cdot)$.

The notion of a first-order approximation, however, requires only a distance function - but not affine linearity. So considering a metric space $(E, d)$ instead of the Euclidean vector space $\mathbb{R}^{N}$, Aubin suggested to introduce a so-called transition $\vartheta:[0,1] \times E \longrightarrow E$. In a word, such a transition determines to which point $\vartheta(h, x) \in E$ the initial point $x \in E$ is moved at time $h \in[0,1]$. If it is sufficiently regular with respect to both arguments, then exactly the same track can be followed in the metric space $(E, d)$ as for ordinary differential equations in $\mathbb{R}^{N}$ - up to the existence theorems of Cauchy-Lipschitz and Nagumo [2,3]. A more detailed summary of this approach is presented in $\S 2$.

In regard to set evolutions, Aubin's typical example is the set $\mathcal{K}\left(\mathbb{R}^{N}\right)$ of nonempty compact subsets of $\mathbb{R}^{N}$ supplied with the classical Pompeiu-Hausdorff metric $d l$ and, transitions are induced by reachable sets of differential inclusions (with bounded and Lipschitz continuous right-hand side).

\section{Obstacles to set evolutions: Boundaries need not evolve continuously.}

A simple attempt of applying Aubin's original theory to first-order set evolutions seems foredoomed to failure. Indeed, consider the example of an annulus expanding isotropically at a constant speed. After a finite period, the "hole" in the center of the annulus is suddenly disappearing.

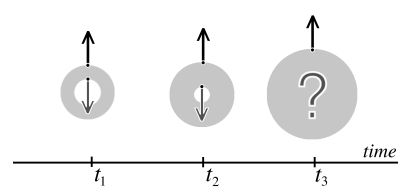
So in particular, the topological boundary of the expanding annulus does not evolve continuously (in the classical sense of Painlevé-Kuratowski). The same phenomenon (of the boundary and its normal cones) causes analytical difficulties also when comparing the evolution of two initial compact sets (see $\S 3.1$ ). This effect has motivated our two main generalizations of Aubin's mutational equations:

Firstly, the basic set $E \neq \emptyset$ is not supplied with a metric (as before), but with a distance function $q: E \times E \longrightarrow[0, \infty[$ satisfying only the triangle inequality and $q(x, x)=0$ for all $x \in E$. In particular, 
$q$ need not be symmetric. Here such a distance function is called ostensible metric. Dispensing with the symmetry of distance functions, we are now free to compare only the normal cones of later sets with the normals of earlier sets - but not necessarily vice versa because roughly speaking, the latter information might have been "lost" in a "disappearing hole" meanwhile. So the ostensible metric on $\mathcal{K}\left(\mathbb{R}^{N}\right)$ used in the main geometric example in $\S 4$ is

$$
q_{\mathcal{K}, N}: \mathcal{K}\left(\mathbb{R}^{N}\right) \times \mathcal{K}\left(\mathbb{R}^{N}\right) \longrightarrow\left[0, \infty\left[, \quad\left(K_{1}, K_{2}\right) \longmapsto d l\left(K_{1}, K_{2}\right)+\operatorname{dist}\left(\operatorname{Graph}{ }^{b} N_{K_{2}} \text {, Graph }{ }^{b} N_{K_{1}}\right)\right.\right.
$$

with $\quad N_{K}(x)$ denoting the limiting normal cone of $K \subset \mathbb{R}^{N}$ at $x \in \partial K$ and

$$
{ }^{b} N_{K}(x):=N_{K}(x) \cap \mathbb{B}_{1}=\left\{v \in N_{K}(x):|v| \leq 1\right\} .
$$

The second aspect is to specify a weaker notion of "solutions". Roughly speaking, distribution-like solutions are introduced in sets without any vector space structure. As the general key idea of distributions, we regard : "Select an important property and then try to preserve it (at least) for all 'test elements' specified before." In the classical sense, this feature is partial integration. For an ostensible metric space $(E, q)$, however, we have chosen the estimate of the distance $h \mapsto q(\vartheta(h, x), \tau(h, y))$ between two transitions $\vartheta, \tau$ starting in any points $x, y \in E$. Now the first point $x$ is to be restricted to a given "test set" $D \subset E$.

These notions lead to so-called forward transitions on an ostensible metric space and right-hand forward solutions of generalized mutational equations. All the details (up to an existence theorem) are presented in $\S \S 3.2,3.3$ and, Aubin's concept of mutational equations proves to be a special case.

\section{The key geometric tool: Reachable sets of differential inclusions — and their regularity.}

All analytical obstacles here result from the geometric effect that "holes" of compact sets might disappear while evolving along differential inclusions. So it plays an important role to specify sufficient conditions on both the initial compact set and the differential inclusion for excluding this effect during a (maybe just very short) period.

Considering nonlinear differential inclusions in $\mathbb{R}^{N}$, investigations about the boundaries of reachable sets are very rare indeed. Bressan's paper [13] exemplifies early results about reachable sets (of a single point) with $C^{1}$ boundary in affine-linear control systems with constant smooth control sets. Recently, Cannarsa and Frankowska published sufficient conditions on control systems for the interior sphere property of their reachable sets [14]. As a consequence, they obtain even sufficient conditions on control system and initial convex set such that its reachable set has $C^{1,1}$ boundary at all small positive times [14, Corollary 3.12]. Independently from them, the author specified other sufficient conditions for preserving a similar property (called positive erosion) of the compact initial set [42].

Further results are presented in the Appendix and to the best of my knowledge, they are new. The proofs here are based on the corresponding Hamilton equations of the boundary trajectories and their adjoints, respectively. In contrast to Rzeżuchowski's papers [51, 52], we draw some conclusions without assuming the Hamiltonian function to be $C^{2}$ and, all our results hold for initial sets consisting of more than a single point.

Proposition 1.1 Let $F: \mathbb{R}^{N} \leadsto \mathbb{R}^{N}$ be a set-valued map satisfying the following conditions:

(i) F has nonempty compact convex values,

(ii) Hamiltonian $\mathcal{H}_{F}(\cdot, \cdot) \in C^{1,1}\left(\mathbb{R}^{N} \times\left(\mathbb{R}^{N} \backslash\{0\}\right)\right)$,

(iii) $\left\|\mathcal{H}_{F}\right\|_{C^{1,1}\left(\mathbb{R}^{N} \times \partial \mathbb{B}_{1}\right)} \stackrel{\text { Def. }}{=}\left\|\mathcal{H}_{F}\right\|_{C^{1}\left(\mathbb{R}^{N} \times \partial \mathbb{B}_{1}\right)}+\left.\operatorname{Lip} D \mathcal{H}_{F}\right|_{\mathbb{R}^{N} \times \partial \mathbb{B}_{1}}<\infty$. 
For every compact $N$-dimensional $C^{1,1}$ submanifold $K$ of $\mathbb{R}^{N}$ with boundary, there exist a time $\tau>0$ and a radius $\rho>0$ such that for all $t \in[0, \tau[$,

1. both $\vartheta_{F}(t, K)$ and its closed complement have positive reach of radius $\geq \rho$.

So in particular, the boundary $\partial \vartheta_{F}(t, K) \subset \mathbb{R}^{N}$ is a $(N-1)$-dimensional $C^{1,1}$ submanifold.

2. $K=\mathbb{R}^{N} \backslash \vartheta_{-F}\left(t, \mathbb{R}^{N} \backslash \vartheta_{F}(t, K)\right)$.

So the evolution of $K$ is "reversible" up to any time $t \in] 0, \tau[$.

Proposition 1.2 Suppose for the set-valued map $F: \mathbb{R}^{N} \leadsto \mathbb{R}^{N}$ :

(i') there is a radius $\rho>0$ such that for each $x \in \mathbb{R}^{N}$, the compact convex set $F(x) \subset \mathbb{R}^{N}$ has the form $F(x)=\mathbb{B}_{\rho}\left(M_{x}\right) \stackrel{\text { Def. }}{=}\left\{y \in \mathbb{R}^{N} \mid \operatorname{dist}\left(y, M_{x}\right) \leq \rho\right\}$ with some $M_{x} \in \mathcal{K}\left(\mathbb{R}^{N}\right)$,

(ii') Hamiltonian $\mathcal{H}_{F}(\cdot, \cdot) \in C^{2}\left(\mathbb{R}^{N} \times\left(\mathbb{R}^{N} \backslash\{0\}\right)\right)$,

(iii) $\left\|\mathcal{H}_{F}\right\|_{C^{1,1}\left(\mathbb{R}^{N} \times \partial \mathbb{B}_{1}\right)} \stackrel{\text { Def. }}{=}\left\|\mathcal{H}_{F}\right\|_{C^{1}\left(\mathbb{R}^{N} \times \partial \mathbb{B}_{1}\right)}+\left.\operatorname{Lip} D \mathcal{H}_{F}\right|_{\mathbb{R}^{N} \times \partial \mathbb{B}_{1}}<\lambda<\infty$.

For any nonempty compact set $K \subset \mathbb{R}^{N}$, there exist a positive constant $\sigma$ and a time $\left.\left.\widehat{\tau} \in\right] 0,1\right]$ (depending only on $\lambda, \rho, K)$ such that for any time $t \in] 0, \widehat{\tau}\left[\right.$ and initial point $x_{0} \in K$, the reachable set $\vartheta_{F}\left(t, x_{0}\right) \subset \mathbb{R}^{N}$ has the form $\vartheta_{F}\left(t, x_{0}\right)=\mathbb{B}_{\sigma t}\left(M_{t, x_{0}}\right)$ with some closed set $M_{t, x_{0}} \subset \mathbb{R}^{N}$.

As an immediate consequence, $\vartheta_{F}\left(t, K_{1}\right)$ has the same property for all times $\left.t \in\right] 0, \widehat{\tau}[$ and each initial subset $K_{1} \in \mathcal{K}\left(\mathbb{R}^{N}\right)$ of $K$.

This paper has the following structure : $\S 2$ is a summary of Aubin's mutational equations in a metric space specifying the terms "transition", "solutions" and stating his main existence theorem (as a counterpart of the Cauchy-Lipschitz theorem about ODEs).

In $\S 3.1$, we specify some more obstacles in detail when applying Aubin's concept to geometric evolutions depending on the topological boundary. They provide the motivation for generalizing mutational equations in $\S 3.2$. After introducing forward transitions, we coin the term of "right-hand forward solutions" of a generalized mutational equation and give sufficient conditions of its existence (corresponding to Peano's theorem about ODEs). In $\S 4$, this concept is applied to compact subsets of the Euclidean space whose evolution is determined by their own nonlocal properties "up to first order".

The appendix provides all the results (and their proofs) about the boundary of reachable sets. 


\section{Ordinary differential equations in metric spaces: A summary of Aubin's mutational equations}

An approach to evolution problems in metric spaces is the mutational analysis of Jean-Pierre Aubin presented in $[2,3]$. It proves to be the more general background of "shape derivatives" introduced by Jean Céa and Jean-Paul Zolésio and has similarities to "quasidifferential equations" of Panasyuk [47].

Roughly speaking, the starting point consists in extending the terms "direction" and "velocity" from vector spaces to metric spaces. Then the basic idea of first-order approximation leads to a definition of derivative for curves in a metric space and step by step, we can follow the same track as for ordinary differential equations.

Let us now describe the mutational approach in more detail : In a vector space like $\mathbb{R}^{N}$, each vector $v \neq 0$. defines a continuous function

$$
\left[0, \infty\left[\times \mathbb{R}^{N} \longrightarrow \mathbb{R}^{N}, \quad(h, x) \longmapsto x+h v\right.\right.
$$

mapping the time $h$ and the initial point $x$ to its final point — similar to the topological notion of a homotopy. This concept does not really require addition or scalar multiplication and thus can be applied to every metric space $(E, d)$ instead :

Definition 2.1 ([2, Aubin 99]) Let $(E, d)$ be a metric space.

$A \operatorname{map} \vartheta:[0,1] \times E \longrightarrow E$ is called transition on $(E, d)$ if it satisfies

1. $\vartheta(0, x)=x \quad$ for all $x \in E$,

2. $\underset{h \downarrow 0}{\limsup } \frac{1}{h} \cdot d(\vartheta(h, \vartheta(t, x)), \vartheta(t+h, x))=0 \quad$ for all $x \in E, t<1$,

3. $\alpha(\vartheta):=\sup _{x \neq y} \limsup _{h \downarrow 0}\left(\frac{d(\vartheta(h, x), \vartheta(h, y))-d(x, y)}{h d(x, y)}\right)^{+}<\infty$,

4. $\beta(\vartheta):=\sup _{x \in E} \limsup _{h \downarrow 0} \frac{1}{h} \cdot d(x, \vartheta(h, x))<\infty$

with the abbreviation $(r)^{+}:=\max (0, r)$ for $r \in \mathbb{R}$.

Condition (1.) guarantees that the second argument $x$ represents the initial point at time $t=0$. Moreover condition (2.) can be regarded as a weakened form of the semigroup property. Finally the parameters $\alpha(\vartheta), \beta(\vartheta)$ imply the continuity of $\vartheta$ with respect to both arguments. In particular, condition (4.) together with Gronwall's Lemma ensures the uniform Lipschitz continuity of $\vartheta$ with respect to time : $\quad d(\vartheta(s, x), \vartheta(t, x)) \leq \beta(\vartheta) \cdot|t-s| \quad$ for all $s, t \in[0,1], x \in E$.

Obviously the function $[0,1] \times \mathbb{R}^{N} \longrightarrow \mathbb{R}^{N},(h, x) \longmapsto x+h v$ mentioned before fulfills the conditions on a transition on $\left(\mathbb{R}^{N},|\cdot|\right)$. Let us give some further examples :

1.) Leaving vector spaces like $\mathbb{R}^{N}$, we consider the set $\mathcal{K}\left(\mathbb{R}^{N}\right)$ of all nonempty compact subsets of $\mathbb{R}^{N}$ supplied with the so-called Pompeiu-Hausdorff distance

$$
d\left(K_{1}, K_{2}\right):=\max \left\{\sup _{x \in K_{1}} \operatorname{dist}\left(x, K_{2}\right), \sup _{y \in K_{2}} \operatorname{dist}\left(y, K_{1}\right)\right\}
$$

It has the advantage that every closed bounded ball in $\left(\mathcal{K}\left(\mathbb{R}^{N}\right), d l\right)$ is compact (see e.g. [2], [49]). Supposing $f: \mathbb{R}^{N} \longrightarrow \mathbb{R}^{N}$ to be bounded and Lipschitz, transitions are defined as reachable sets of the vector field $f$,

$$
\begin{aligned}
\vartheta_{f}:[0,1] \times \mathcal{K}\left(\mathbb{R}^{N}\right) & \longrightarrow \mathcal{K}\left(\mathbb{R}^{N}\right) \\
\left(t, K_{0}\right) & \longmapsto\left\{x(t) \mid \exists x(\cdot) \in C^{1}\left([0, t], \mathbb{R}^{N}\right): \frac{d}{d t} x(\cdot)=f(x(\cdot)), x(0) \in K_{0}\right\} .
\end{aligned}
$$


The Theorem of Cauchy-Lipschitz ensures that $\vartheta_{f}$ is a transition on $\left(\mathcal{K}\left(\mathbb{R}^{N}\right), d\right)$ and, $\alpha\left(\vartheta_{f}\right) \leq$ Lip $f, \beta\left(\vartheta_{f}\right) \leq\|f\|_{L^{\infty}}$ (see [2, Proposition 3.5.2]). In this regard, we find a close relation to the velocity method of Céa et al. in shape optimization.

2.) Now more than one velocity is admitted at every point of $\mathbb{R}^{N}$, i.e. strictly speaking, we consider the differential inclusion $\frac{d}{d t} x(\cdot) \in F(x(\cdot))$ (a.e.) with a set-valued map $F: \mathbb{R}^{N} \leadsto \mathbb{R}^{N}$ instead of the ODE $\frac{d}{d t} x(\cdot)=f(x(\cdot))$. For every bounded Lipschitz map $F: \mathbb{R}^{N} \leadsto \mathbb{R}^{N}$ with convex values in $\mathcal{K}\left(\mathbb{R}^{N}\right)$,

$$
\begin{aligned}
& \vartheta_{F}:[0,1] \times \mathcal{K}\left(\mathbb{R}^{N}\right) \longrightarrow \mathcal{K}\left(\mathbb{R}^{N}\right) \\
&\left(t, K_{0}\right) \longmapsto\left\{x(t) \mid \exists x(\cdot) \in W^{1,1}\left([0, t], \mathbb{R}^{N}\right):\right. \\
&\left.\frac{d}{d t} x(\cdot) \in F(x(\cdot)) \text { a.e. } \quad x(0) \in K_{0}\right\}
\end{aligned}
$$

is a transition on $\left(\mathcal{K}\left(\mathbb{R}^{N}\right), d l\right)$ - as a consequence of Filippov's Theorem (see [2, Aubin 99: Proposition 3.7.3]). For any $\lambda>0, \quad \operatorname{LIP}_{\lambda}\left(\mathbb{R}^{N}, \mathbb{R}^{N}\right)$ abbreviates the set of bounded $\lambda$-Lipschitz continuous maps $F: \mathbb{R}^{N} \leadsto \mathbb{R}^{N}$ with nonempty compact convex values.

In contrast to example (1.), the reachable set $\vartheta_{F}\left(t, K_{0}\right)$ of a set-valued map $F$ might change its topological properties. $F(\cdot):=$ $\mathbb{B}_{1} \stackrel{\text { Def. }}{=}\left\{v \in \mathbb{R}^{N}|| v \mid \leq 1\right\}$, for example, leads to the expansion with constant speed 1 in all directions and makes the "hole" of the annulus $K_{0}:=\{x|1 \leq| x \mid \leq 2\} \subset \mathbb{R}^{N}$ disappear at time 1 .

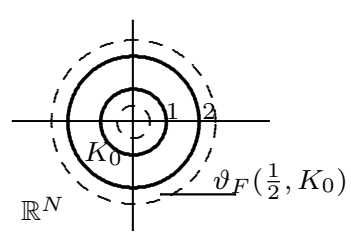

This effect cannot occur in the examples of ordinary differential equations (with Lipschitz righthand side) since their evolutions are reversible in time.

A transition $\vartheta:[0,1] \times E \longrightarrow E$ provides a first-order approximation of a curve $x(\cdot):[0, T[\longrightarrow E$ at time $t \in\left[0, T\left[\right.\right.$ if $\quad \limsup _{h \downarrow 0} \frac{1}{h} \cdot d(\vartheta(h, x(t)), x(t+h))=0$.

Naturally $\vartheta$ need not be unique in general and thus,

Definition 2.2 Let $(E, d)$ be a metric space and $x(\cdot):[0, T[\longrightarrow E$ a curve.

The so-called mutation of $x(\cdot)$ at time $t \in[0, T[$, abbreviated as $\stackrel{\circ}{x}(t)$, consists of all transitions $\vartheta$ satisfying $\limsup _{h \downarrow 0} \frac{1}{h} \cdot d(\vartheta(h, x(t)), \quad x(t+h))=0$.

Remark 2.3 As an immediate consequence of Definition 2.1 (2), every transition $\vartheta$ belongs to its own mutation in the sense of $\vartheta \in \stackrel{\circ}{x}(t)$ for $x(\cdot):=\vartheta\left(\cdot, x_{0}\right)$ with any $x_{0} \in E, t \in[0,1[$.

A mutational equation is based on a given function $f$ of time $t \in[0, T[$ and state $x \in E$ whose values are transitions on $(E, d)$, i.e. $\quad f: E \times[0, T[\longrightarrow \Theta(E, d),(x, t) \longmapsto f(x, t), \quad$ and, we look for a Lipschitz curve $x(\cdot):[0, T[\longrightarrow(E, d)$ such that $f(x(t), t)$ belongs to its mutation $\stackrel{\circ}{x}(t)$ for almost every time $t \in[0, T[$ (see [2, Definition 1.3.1]).

The Theorem of Cauchy-Lipschitz and its proof suggest Euler method for constructing solutions of mutational equations. In this context we need an upper estimate of the distance between two points while evolving along two (different) transitions.

First of all, a distance between two transitions $\vartheta, \tau:[0,1] \times E \longrightarrow E$ has to be defined and, it is based on comparing the evolution of one and the same initial point : 
Definition 2.4 ([2, Definition 1.1.2]) Let $(E, d)$ be a metric space. For any two transitions $\vartheta, \tau$ on $(E, d)$, define $\quad D(\vartheta, \tau):=\sup _{x \in E} \limsup _{h \downarrow 0} \frac{1}{h} \cdot d(\vartheta(h, x), \tau(h, x))$.

As an immediate consequence of triangle inequality, $D(\vartheta, \tau) \leq \beta(\vartheta)+\beta(\tau)<\infty$.

Considering the preceding example of $\left(\mathcal{K}\left(\mathbb{R}^{N}\right), d l\right)$ and reachable sets $\vartheta_{F}, \vartheta_{G}$ of bounded Lipschitz maps $F, G: \mathbb{R}^{N} \leadsto \mathbb{R}^{N}$, Filippov's Theorem implies $D\left(\vartheta_{F}, \vartheta_{G}\right) \leq \sup _{x \in \mathbb{R}^{N}} d l(F(x), G(x)) \quad($ see $[2$, Proposition 3.7.3]). In general, these definitions lead to the substantial estimate :

Lemma 2.5 ([2, Lemma 1.1.3]) For any transitions $\vartheta, \tau$ on a metric space $(E, d)$ and initial points $x, y \in E$, the distance at each time $h \in[0,1]$ satisfies

$$
d(\vartheta(h, x), \tau(h, y)) \leq d(x, y) \cdot e^{\alpha(\vartheta) h}+h D(\vartheta, \tau) \cdot \frac{e^{\alpha(\vartheta) h}-1}{\alpha(\vartheta) h} .
$$

The proof of this inequality provides an excellent insight into the basic technique for drawing global conclusions from local properties : Due to the definition of transitions, the distance $\psi:[0,1] \longrightarrow[0, \infty[$, $h \longmapsto d(\vartheta(h, x), \tau(h, y))$ is a Lipschitz continuous function of time and satisfies

$$
\begin{aligned}
& \lim _{h \downarrow 0} \frac{\psi(t+h)-\psi(t)}{h}=\lim _{h \downarrow 0} \quad \frac{1}{h} \cdot(d(\vartheta(t+h, x), \quad \tau(t+h, y)) \quad-d(\vartheta(t, x), \tau(t, y))) \\
& \leq \limsup _{h \downarrow 0} \frac{1}{h} \cdot(d(\vartheta(t+h, x), \quad \vartheta(h, \vartheta(t, x)))+ \\
& d(\vartheta(h, \vartheta(t, x)), \vartheta(h, \tau(t, y)))-d(\vartheta(t, x), \tau(t, y))+ \\
& d(\vartheta(h, \tau(t, y)), \tau(h, \tau(t, y)))+ \\
& d(\tau(h, \tau(t, y)), \tau(t+h, \quad y))) \\
& \leq 0+\alpha(\vartheta) \cdot \psi(t)+D(\vartheta, \tau)+0
\end{aligned}
$$

for almost every $t \in[0,1$ [ (i.e. every $t$ at which the limit on the left-hand side exists). So the estimate results from well-known Gronwall's Lemma about Lipschitz continuous functions. In fact, Gronwall's Lemma proves to be the key analytical tool for all these conclusions of mutational analysis and, its integral version holds even for continuous functions (see [2, Lemma 8.3.1]).

Considering now mutational equations, Lemma 2.5 is laying the foundations for proving the convergence of Euler method. It leads to the following mutational counterpart of the Theorem of CauchyLipschitz (quoted from [2, Aubin 99: Theorem 1.4.2]) - ensuring existence, uniqueness as well as continuity with respect to the right-hand side.

Theorem 2.6 ([2]) Assume that the closed bounded balls of the metric space (E, d) are compact. Let $f$ be a function from $E$ to a set $\Theta(E, d)$ of transitions on $(E, d)$ satisfying

1. $\exists \lambda>0: D(f(x), f(y)) \leq \lambda \cdot d(x, y) \quad$ for all $x, y \in E$

2. $A:=\sup _{x \in E} \alpha(f(x))<\infty$.

Suppose for $y:[0, T[\longrightarrow$ that its mutation $\stackrel{\circ}{y}(t)$ is nonempty for each $t$.

Then for every initial value $x_{0} \in E$, there exists a unique solution $x(\cdot):[0, T[\longrightarrow E$ of the mutational equation $\stackrel{\circ}{x}(t) \ni f(x(t))$, i.e. $x(\cdot)$ is Lipschitz continuous and for almost every $t \in[0, T[$,

$$
\limsup _{h \downarrow 0} \frac{1}{h} \cdot d(x(t+h), f(x(t))(h, x(t)))=0,
$$

satisfying, in addition, $x(0)=x_{0}$ and the inequality (for every $t \in[0, T[$ )

$$
d(x(t), y(t)) \leq d\left(x_{0}, y(0)\right) \cdot e^{(A+\lambda) t}+\int_{0}^{t} e^{(A+\lambda)(t-s)} \cdot \inf _{\vartheta \in \grave{y}(s)} D(f(y(s)), \vartheta) d s .
$$




\section{Generalizing mutational equations : Nonsymmetric distance and distribution-like solutions.}

\subsection{Obstacles to first-order geometric evolutions due to boundaries}

Applying the mutational analysis of Aubin to a metric space $(E, d)$, obstacles are mostly related to the continuity parameters of a transition $\vartheta$

$$
\begin{array}{llll}
\alpha(\vartheta) \stackrel{\text { Def. }}{=} & \sup _{x \neq y} \limsup _{h \downarrow 0}\left(\frac{d(\vartheta(h, x), \vartheta(h, y))-d(x, y)}{h d(x, y)}\right)^{+}<\infty, \\
\beta(\vartheta) \stackrel{\text { Def. }}{=} & \sup _{x \in E} \limsup _{h \downarrow 0} \frac{1}{h} \cdot d(x, \vartheta(h, x)) & <\infty .
\end{array}
$$

In regard to first-order geometric evolutions, these difficulties arise when incorporating normal cones into a distance function of compact subsets. We are going to use reachable sets $\vartheta_{F}(\cdot, \cdot)$ of differential inclusions $\dot{x}(\cdot) \in F(x(\cdot))$ a.e. as candidates for transitions on $\mathcal{K}\left(\mathbb{R}^{N}\right)$. So the topological properties of $\vartheta_{F}(t, K)$ may change in the course of time.

\section{For the regularity in time : Ostensible metrics}

Let us consider first the consequences of the boundary for the continuity of $\vartheta_{F}:[0,1] \times \mathcal{K}\left(\mathbb{R}^{N}\right) \longrightarrow$ $\mathcal{K}\left(\mathbb{R}^{N}\right)$ with respect to time.

The key aspect is illustrated easily by an annulus $K_{\odot}$ expanding isotropically at a constant speed. After a positive finite time $t_{3}$, the "hole" in the center has disappeared of course.

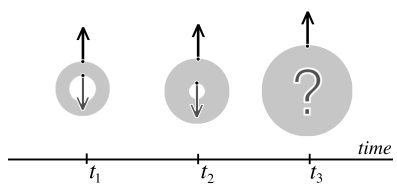

In general, the topological boundary of $\vartheta_{F}(\cdot, K):\left[0, \infty\left[\leadsto \mathbb{R}^{N}\right.\right.$ (with $K \in \mathcal{K}\left(\mathbb{R}^{N}\right)$ ) is not continuous with respect to $\boldsymbol{d}$. Furthermore, the normals of later sets find close counterparts among the normals of earlier sets, but usually not vice versa. So we dispense with the symmetry condition on a metric :

Definition 3.1 Let $E$ be a nonempty set. $q: E \times E \longrightarrow[0, \infty[$ is called ostensible metric on $E$ if it satisfies the conditions :
1. $\forall x \in E$ :
$q(x, x)=0$
(reflexive)
2. $\forall x, y, z \in E: \quad q(x, z) \leq q(x, y)+q(y, z)$
(triangle inequality).

Then $(E, q)$ is called ostensible metric space.

In the literature on topology (e.g. [55, Wilson 31], [33, Kelly 63], [53, Stoltenberg 69], [35, Künzi $92]$ ), a quasi-metric $p: E \times E \longrightarrow[0, \infty[$ on a set $E$ satisfies the triangle inequality and is positive definite, i.e. $p(x, y)=0 \Longleftrightarrow x=y$ for every $x, y \in E$. A pseudo-metric $p: E \times E \longrightarrow[0, \infty[$ on a set $E \neq \emptyset$ is characterized by the properties : reflexive (i.e. $p(x, x)=0$ for all $x$ ), symmetric (i.e. $p(x, y)=p(y, x)$ for all $x, y)$ and the triangle inequality. So this generalized distance of Definition 3.1 is sometimes called quasi-pseudo-metric (see [33, Kelly 63], [35, Künzi 92], for example), but just for linguistic reasons we prefer the adjective "ostensible". 
For the regularity with respect to initial states : the distributional notion

Applying now the steps of mutational analysis to an ostensible metric space $(E, q)$, we encounter analytical obstacles soon. In particular, $\quad[0,1] \longrightarrow\left[0, \infty\left[, \quad t \longmapsto q\left(\vartheta\left(t, x_{1}\right), \vartheta\left(t, x_{2}\right)\right) \quad\right.\right.$ need not be continuous for arbitrary initial elements $x_{1}, x_{2} \in E$.

Consider, for example, reachable sets $\vartheta_{F}\left(t, K_{1}\right), \vartheta_{F}\left(t, K_{2}\right)$ of an autonomous differential inclusion $\dot{x}(\cdot) \in F(x(\cdot))$ with initial sets $K_{1}, K_{2} \in \mathcal{K}\left(\mathbb{R}^{N}\right)$ and a given map $F \in \operatorname{LIP}_{\lambda}\left(\mathbb{R}^{N}, \mathbb{R}^{N}\right)$. The figure on the right-hand side sketches a situation in which the distance between topological boundaries $t \longmapsto \operatorname{dist}\left(\partial K_{2}, \partial K_{1}\right)$ cannot be continuous. So even if we do not take normal cones into account, it is difficult to find an ostensible metric on $\mathcal{K}\left(\mathbb{R}^{N}\right)$ depending on the boundary, but without such a lack of continuity.

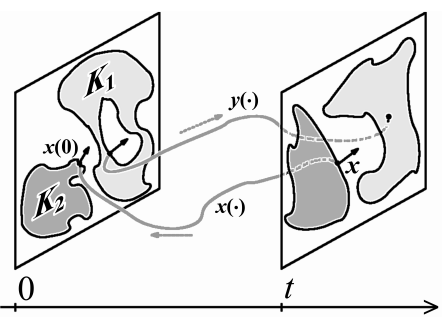

As a first important consequence, we cannot apply the proof of key estimate $(*)$ (in Lemma 2.5) to ostensible metric spaces immediately. A more general form of Gronwall's Lemma is needed instead supposing semicontinuity (rather than continuity). Its easy indirect proof is presented in [41, 43].

When extending key estimate $(*)$ to transitions $\vartheta, \tau$ on an ostensible metric space $(E, q)$, the required semicontinuity of $t \longmapsto q(\vartheta(t, x), \tau(t, y))$ will be guaranteed by a further condition on generalized transitions.

\section{Lemma 3.2 (Lemma of Gronwall for semicontinuous functions I)}

Let $\psi:[a, b] \longrightarrow \mathbb{R}, f, g \in C^{0}([a, b[, \mathbb{R})$ satisfy $f(\cdot) \geq 0$ and

$$
\begin{aligned}
\psi(t) & \leq \limsup _{h \downarrow 0} \psi(t-h) & & \text { for all } t \in] a, b], \\
\psi(t) & \geq \limsup _{h \downarrow 0} \psi(t+h) & & \text { for all } t \in[a, b[, \\
\limsup _{h \downarrow 0} \frac{\psi(t+h)-\psi(t)}{h} & \leq f(t) \cdot \limsup _{h \downarrow 0} \psi(t-h)+g(t) & & \text { for all } t \in] a, b[.
\end{aligned}
$$

Then, for every $t \in[a, b]$, the function $\psi(\cdot)$ fulfills the upper estimate

$$
\psi(t) \leq \psi(a) \cdot e^{\mu(t)}+\int_{a}^{t} e^{\mu(t)-\mu(s)} g(s) d s \quad \text { with } \mu(t):=\int_{a}^{t} f(s) d s .
$$

Remark 3.3 (i) The condition $\limsup _{h \downarrow 0} \frac{\psi(t+h)-\psi(t)}{h} \leq f(t) \cdot \psi(t)+g(t)$ (supposed in the widespread forms of Gronwall's Lemma) is stronger than the third assumption of this lemma due to the semicontinuity condition $\psi(t) \leq \limsup _{h \downarrow 0} \psi(t-h)$.

(ii) This and the following subdifferential version of Gronwall's Lemma also hold if the functions $f, g:[a, b[\longrightarrow \mathbb{R}$ are only upper semicontinuous (instead of continuous). The proof is based on upper approximations of $f(\cdot), g(\cdot)$ by continuous functions.

Corollary 3.4 (Lemma of Gronwall for semicontinuous functions II)

Let $\psi:[a, b] \longrightarrow \mathbb{R}, f, g \in C^{0}([a, b[, \mathbb{R})$ satisfy $f(\cdot) \geq 0$ and

$$
\begin{aligned}
\psi(t) & \leq \liminf _{h \downarrow 0} \psi(t-h) & & \text { for all } t \in] a, b], \\
\psi(t) & \geq \liminf _{h \downarrow 0} \psi(t+h) & & \text { for all } t \in[a, b[, \\
\liminf _{h \downarrow 0} \frac{\psi(t+h)-\psi(t)}{h} & \leq f(t) \cdot \liminf _{h \downarrow 0} \psi(t-h)+g(t) & & \text { for all } t \in] a, b[.
\end{aligned}
$$
Then, for every $t \in[a, b], \quad \psi(t) \leq \psi(a) \cdot e^{\mu(t)}+\int_{a}^{t} e^{\mu(t)-\mu(s)} g(s) d s \quad$ with $\mu(t):=\int_{a}^{t} f(s) d s$ 
Nevertheless, we have to exclude such a discontinuity of evolving boundaries - for short times at least. In the geometric example about reachable sets, additional assumptions about $K_{1}$ are needed. Suitable conditions on $F$ can guarantee that compact sets with $C^{1,1}$ boundary preserve this regularity for short times (see Appendix A.2) and, their topological properties do not change. Assuming further conditions on one of the sets $K_{1}, K_{2} \in \mathcal{K}\left(\mathbb{R}^{N}\right)$ prevents us from applying the mutational analysis of Aubin, though. So, we use the basic idea of distributions.

In an ostensible metric space, there are no obvious generalizations of linear forms or partial integration and so, distributions in their widespread sense cannot be introduced. More generally speaking, however, their basic idea is to select an important property and demand it for all elements of a given "test set".

In the mutational analysis of a metric space $(E, d)$, the estimate of Lemma 2.5

$$
d(\vartheta(h, x), \tau(h, y)) \leq d(x, y) \cdot e^{\alpha(\vartheta) h}+h D(\vartheta, \tau) \cdot \frac{e^{\alpha(\vartheta) h}-1}{\alpha(\vartheta) h}
$$

(for arbitrary $x, y \in E$ and $h \in[0,1[$ ) represents the probably most important tool for constructing solutions by means of Euler method. So it is our starting point for overcoming the recent obstacle, i.e. we are interested in how to realize the formal estimate

$$
q(\vartheta(h, z), \tau(h, y)) \leq\left(q(z, y)+h Q^{\mapsto}(\vartheta, \tau)\right) \cdot e^{\alpha^{\mapsto} h}
$$

for all points $y \in E$, every element $z$ of a given "test set" $D \subset E$ and $h>0$ sufficiently small (depending only on $\vartheta, z)$. In particular, the definitions of $Q^{\mapsto}(\vartheta, \tau)$ and the parameter $\alpha^{\mapsto}$ have to be adapted.

\subsection{Forward transitions}

From now on, let $E$ denote a nonempty set and fix $D \subset E$ as set of "test elements" (for later comparisons). Furthermore suppose $q: E \times E \longrightarrow[0, \infty[$ to be an ostensible metric on $E$.

Now we specify the primary tools for describing deformations in the tuple $(E, D, q)$. A map $\vartheta:[0,1] \times$ $E \longrightarrow E$ is to define which point $\vartheta(t, x) \in E$ is reached from the initial point $x \in E$ after time $t$. Of course, $\vartheta$ has to fulfill some regularity conditions so that it may form the basis for a calculus of differentiation.

Definition 3.5 A map $\vartheta:[0,1] \times E \longrightarrow E$ is a so-called forward transition on $(E, D, q)$ if it fulfills the following conditions

1. $\vartheta(0, \cdot)=\operatorname{Id}_{E}$,

2. $\forall x \in E, t \in[0,1[:$

$\forall x \in E, t \in[0,1[:$

3. $\exists \alpha^{\mapsto}(\vartheta)<\infty \quad \forall z \in D, y \in E:$

$\begin{array}{ll}\limsup _{h \downarrow 0} & \frac{1}{h} \cdot q(\vartheta(h, \vartheta(t, x)), \quad \vartheta(t+h, x))=0 \\ \underset{h \downarrow 0}{\limsup } & \frac{1}{h} \cdot q(\vartheta(t+h, x), \quad \vartheta(h, \vartheta(t, x)))=0\end{array}$

$\forall, z \in D, y \in E:$

4. $\exists \beta(\vartheta)<\infty \quad \forall s<t \leq 1, x \in E$ :

$\limsup _{h \downarrow 0}\left(\frac{q(\vartheta(h, z), \vartheta(h, y))-q(z, y)}{h}\right)^{+} \leq \alpha^{\mapsto}(\vartheta) q(z, y)$

5. $\forall z \in D$

$\left.\left.\exists \mathcal{T}_{\Theta}=\mathcal{T}_{\Theta}(\vartheta, z) \in\right] 0,1\right]:$

$q(\vartheta(s, x), \vartheta(t, x)) \leq \beta(\vartheta) \cdot(t-s)$

6. $\left.\forall z \in D, y \in E, t \in] 0, \mathcal{T}_{\Theta}\right]$ :

$\left\{\vartheta(t, z) \mid t \in\left[0, \mathcal{T}_{\Theta}\right]\right\} \subset D$,

$\limsup _{h \downarrow 0} q(\vartheta(t-h, z), y) \geq q(\vartheta(t, z), y)$

Here the term "forward" and the symbol $\mapsto$ (representing the time axis) indicate that we usually compare the state at time $t$ with the element at time $t+h$ for $h \downarrow 0$.

Condition (2.) can be regarded as a weakened form of the semigroup property. It consists of two demands as $q$ need not be symmetric. Condition (3.) concerns the continuity properties of $\vartheta$ with 
respect to the initial point. In particular, the first argument of $q$ is restricted to elements $z$ of the "test set" $D$ and, $\alpha^{\mapsto}(\vartheta)$ may be chosen larger than necessary. Thus, it is easier to define $\alpha^{\mapsto}(\cdot)<\infty$ uniformly in some applications like the first-order geometric example of $\S 4$. In condition (4.), all $\vartheta(\cdot, x):[0,1] \longrightarrow E(x \in E)$ are supposed to be equi-Lipschitz continuous.

Condition (5.) guarantees that every element $z \in D$ stays in the "test set" $D$ for short times at least. This assumption is required because estimates using the parameter $\alpha^{\mapsto}(\cdot)$ can be ensured only within this period. Further conditions on $\mathcal{T}_{\Theta}(\vartheta, \cdot)>0$ are avoidable for proving existence of solutions, but they are used for uniqueness.

Condition (6.) forms the basis for applying Gronwall's Lemma 3.2. Indeed, every function $y:[0,1] \longrightarrow E$ with $q(y(t-h), y(t)) \longrightarrow 0$ (for $h \downarrow 0$ and each $t$ ) satisfies

$$
q(\vartheta(t, z), y(t)) \leq \limsup _{h \downarrow 0} q(\vartheta(t-h, z), y(t-h))
$$

for all elements $z \in D$ and times $\left.t \in] 0, \mathcal{T}_{\Theta}(\vartheta, x)\right]$.

Definition 3.6 $\quad \Theta^{\mapsto}(E, D, q)$ denotes a set of forward transitions on $(E, D, q)$ supposing for all its elements $\vartheta, \tau \in \Theta^{\mapsto}(E, D, q)$,

$$
Q^{\mapsto}(\vartheta, \tau):=\sup _{z \in D, y \in E} \limsup _{h \downarrow 0} \frac{1}{h} \cdot\left(q(\vartheta(h, z), \tau(h, y))-q(z, y) \cdot e^{\alpha^{\mapsto}(\tau) h}\right)^{+}<\infty .
$$

These definitions enable us to compare any element $y \in E$ with a "test element" $z \in D$ while evolving along two forward transitions. Considering the bound in the next proposition, the influence of the distances between initial points and between transitions is the same as for ordinary differential equations. The key idea of right-hand forward solutions has been to preserve this structural estimate while extending mutational equations to ostensible metrics and "distributional" features (in regard to a test set $D)$.

Proposition 3.7 Let $\vartheta, \tau \in \Theta^{\mapsto}(E, D, q)$ be forward transitions, $z \in D, y \in E$ and $0 \leq t_{1} \leq t_{2} \leq 1$, $h \geq 0$ satisfying $t_{1}+h<\mathcal{T}_{\Theta}(\vartheta, z)$. Then,

$$
q\left(\vartheta\left(t_{1}+h, z\right), \tau\left(t_{2}+h, y\right)\right) \leq\left(q\left(\vartheta\left(t_{1}, z\right), \tau\left(t_{2}, y\right)\right)+h \cdot Q^{\mapsto}(\vartheta, \tau)\right) \cdot e^{\alpha^{\mapsto}(\tau) h} .
$$

Proof. The auxiliary function $\varphi: h \longmapsto q\left(\vartheta\left(t_{1}+h, z\right), \tau\left(t_{2}+h, y\right)\right)$ has the semicontinuity property $\varphi(h) \leq \limsup _{k \downarrow 0} \quad \varphi(h-k)$ due to the assumptions of $\Theta^{\mapsto}(E, D, q)$.

Moreover it fulfills $\quad \limsup _{k \downarrow 0} \frac{\varphi(h+k)-\varphi(h)}{k} \leq \alpha^{\mapsto}(\tau) \cdot \varphi(h)+Q^{\mapsto}(\vartheta, \tau) \quad$ for any $h \in[0,1[$ with $t_{1}+h<\mathcal{T}_{\Theta}(\vartheta, z)$. Indeed, for all $k>0$ sufficiently small, the triangle inequality leads to

$$
\begin{aligned}
& \varphi(h+k) \leq q\left(\vartheta\left(t_{1}+h+k, z\right), \quad \vartheta\left(k, \vartheta\left(t_{1}+h, z\right)\right)\right) \\
& +q\left(\vartheta\left(k, \vartheta\left(t_{1}+h, z\right)\right), \quad \tau\left(k, \tau\left(t_{2}+h, y\right)\right)\right) \\
& +q\left(\tau\left(k, \tau\left(t_{2}+h, y\right)\right), \quad \tau\left(t_{2}+h+k, \quad y\right)\right) \\
& \leq \quad 0+Q^{\mapsto}(\vartheta, \tau) \cdot k+\varphi(h) e^{\alpha^{\natural}(\tau) k}+0+o(k)
\end{aligned}
$$

since $t_{1}+h+k<\mathcal{T}_{\Theta}(\vartheta, z)$ implies $\vartheta\left(t_{1}+h, z\right), \vartheta\left(t_{1}+h+k, z\right) \in D$.

Thus the claim results from Gronwall's Lemma 3.2. 


\subsection{Right-hand forward solutions}

The next step is to define the term "right-hand forward primitive" for a curve $\vartheta(\cdot):[0, T] \longrightarrow$ $\Theta^{\mapsto}(E, D, q)$ of forward transitions.

Roughly speaking, a curve $x(\cdot):[0, T[\longrightarrow E$ represents a primitive of $\vartheta(\cdot)$ if at each time $t \in[0, T[$, the forward transition $\vartheta(t)$ can be interpreted as a first-order approximation of $x(t+\cdot)$. Combining this notion with the key estimate of Proposition 3.7, a vague meaning of "first-oder approximation" is provided : Comparing $x(t+\cdot$ ) with $\vartheta(t)(\cdot, z)$ (for any test element $z \in D$ ), the same estimate ought to hold as if the factor $Q^{\mapsto}(\cdot, \cdot)$ was 0 . It motivates the following definition with the expression "right-hand" indicating that $x(\cdot)$ appears in the second argument of the distances $q$ in condition (1.).

Definition 3.8 The curve $x(\cdot):[0, T[\longrightarrow(E, q)$ is called right-hand forward primitive of a map $\vartheta(\cdot):\left[0, T\left[\Theta^{\mapsto}(E, D, q)\right.\right.$, abbreviated to $\stackrel{\circ}{x}(\cdot) \ni \vartheta(\cdot)$, if

1. $\forall t \in[0, T[\quad \exists \widehat{\alpha}(t) \in[0, \infty[:$

$\limsup _{h \downarrow 0} \frac{1}{h} \cdot\left(q(\vartheta(t)(h, z), x(t+h))-q(z, x(t)) \cdot e^{\widehat{\alpha}(t) \cdot h}\right) \leq 0 \quad$ for every element $z \in D$,

2. $x(\cdot)$ is uniformly continuous in time direction with respect to q, i.e. $\exists \omega(\cdot):] 0, T[\longrightarrow[0, \infty[$ such that $\limsup _{h \downarrow 0} \omega(h)=0 \quad$ and $\quad q(x(s), x(t)) \leq \omega(t-s) \quad$ for $0 \leq s<t<T$.

Remark 3.9 Forward transitions induce their own primitives. To be more precise, every constant function $\vartheta(\cdot):\left[0,1\left[\longrightarrow \Theta^{\mapsto}(E, D, q)\right.\right.$ with $\vartheta(\cdot)=\vartheta_{0}$ has the right-hand forward primitives $[0,1[\longrightarrow E$, $t \longmapsto \vartheta_{0}(t, x)$ with any $x \in E-$ as an immediate consequence of Proposition 3.7. This property is easy to extend to piecewise constant functions $\left[0, T\left[\longrightarrow \Theta^{\mapsto}(E, D, q)\right.\right.$ and so it forms the basis for Euler approximations.

Definition 3.10 For $f: E \times\left[0, T\left[\longrightarrow \Theta^{\mapsto}(E, D, q)\right.\right.$ given, a map $x:[0, T[\longrightarrow E$ is a right-hand forward solution of the generalized mutational equation $\stackrel{\circ}{x}(\cdot) \ni f(x(\cdot), \cdot)$ if $x(\cdot)$ is right-hand forward primitive of $f(x(\cdot), \cdot):\left[0, T\left[\longrightarrow \Theta^{\mapsto}(E, D, q)\right.\right.$.

Constructing solutions of ordinary differential equations is usually based on completeness or compactness. Here we prefer sequential compactness since the available estimates for transitions on $(E, D, q)$ hold only for elements of $D$ in the first argument of $q$ (as in Proposition 3.7). So there is no obvious way of verifying the assumptions of Banach's contraction principle in $(E, q)$.

In Aubin's mutational analysis on metric spaces, bounded closed balls are supposed to be compact, i.e. for every bounded sequence $\left(x_{n}\right)_{n \in \mathbb{N}}$ in $(E, d)$, there exist a subsequence $\left(x_{n_{j}}\right)_{j \in \mathbb{N}}$ and an element $x \in E$ with $d\left(x_{n_{j}}, x\right) \longrightarrow 0$ (for $j \longrightarrow \infty$ ). Dispensing now with the symmetry of the distance, sequential compactness is to consist of two conditions.

Definition 3.11 $(E, q)$ is called two-sided sequentially compact if for any sequence $\left(x_{n}\right)_{n \in \mathbb{N}}$ in $E$ with $\sup _{n} q\left(x_{1}, x_{n}\right)<\infty$, there exist a subsequence $\left(x_{n_{j}}\right)_{j \in \mathbb{N}}$ and an element $x \in E$ such that

$$
q\left(x_{n_{j}}, x\right) \longrightarrow 0, \quad q\left(x, x_{n_{j}}\right) \longrightarrow 0 \quad \text { for } j \longrightarrow \infty .
$$

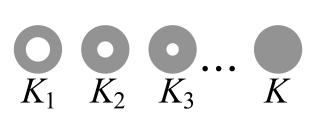

Some ostensible metric spaces have this (rather local) compactness property in common like $\left(\mathcal{K}\left(\mathbb{R}^{N}\right), d l\right)$, but in general, it is too restrictive. Consider e.g. $K_{n}:=$ $\left\{\frac{1}{n+1} \leq|x| \leq 1\right\} \quad$ and $K:=\mathbb{B}_{1} \quad$ satisfying $\quad d\left(K_{n}, K\right)+\operatorname{dist}\left(\partial K, \partial K_{n}\right) \longrightarrow 0$ $(n \rightarrow \infty)$, but $\operatorname{dist}\left(\partial K_{n}, \partial K\right) \geq \frac{1}{2}$. 
For this reason, we coin a more general term of sequential compactness. It is particularly adapted to the aspects of Euler approximations as they are specified for proving the existence of solutions afterwards. Furthermore, considering vanishing "perturbations in time" (here $y_{n}\left(h_{j}\right)$ ) will prove to be essential in the main geometric example of $\S 4$ about reachable sets of differential inclusions (see Proposition 4.17) because the differential inclusions there will have "smoothening" effects on arbitrary boundaries (even after an arbitrarily short period).

Definition 3.12 Let $\Theta$ denote a nonempty subset of forward transitions on $(E, D, q)$.

The tuple $(E, q, \Theta)$ is called transitionally compact if it has the property:

Let the sequences $\left(x_{n}\right)_{n \in \mathbb{N}}$ in $E,\left(h_{j}\right)_{j \in \mathbb{N}}$ in $] 0,1\left[\right.$ and $\left(\vartheta_{n}(\cdot)\right)_{n \in \mathbb{N}}$ in $[0,1] \longrightarrow \Theta$ satisfy

1.) $\sup _{n} q\left(x_{0}, x_{n}\right)<\infty$,

2.) $h_{j} \longrightarrow 0$ for $j \rightarrow \infty$,

3.) $\sup _{n, t} \beta\left(\vartheta_{n}(t)\right)<\infty$,

4.) each $\vartheta_{n}(\cdot):[0,1] \longrightarrow \Theta$ is piecewise constant, i.e. for each $n \in \mathbb{N}$, there exists a finite partiction $0=s_{n, 0}<s_{n, 1}<\ldots<s_{n, k_{n}}=1$ such that $\vartheta_{n}(\cdot)$ is constant in each $\left[s_{n, i}, s_{n, i+1}[\right.$.

For each $n \in \mathbb{N}$, define the function $y_{n}(\cdot):[0,1] \longrightarrow E$ with $y_{n}(0):=x_{n}$ in the piecewise way as $y_{n}(t):=\vartheta_{n}\left(s_{n, i}\right)\left(t-s_{n, i}, y_{n}\left(s_{n, i}\right)\right)$ for all $\left.\left.t \in\right] s_{n, i}, s_{n, i+1}\right]$.

Then there exist a sequence $n_{k} \nearrow \infty$ of indices and $x \in E$ satisfying

$$
\begin{aligned}
& \limsup _{k \longrightarrow \infty} q\left(x_{n_{k}}, x\right) \quad=0, \\
& \limsup _{j \longrightarrow \infty} \sup _{k \geq j} q\left(x, \quad y_{n_{k}}\left(h_{j}\right)\right)=0 .
\end{aligned}
$$

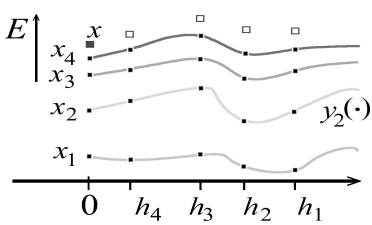

A nonempty subset $F \subset E$ is called transitionally compact in $(E, q, \Theta)$ if the same property holds for any sequence $\left(x_{n}\right)_{n \in \mathbb{N}}$ in $F$ (but $x \in F$ is not required).

Remark 3.13 If $(E, q)$ is two-sided sequentially compact, then $(E, q, \Theta)$ is transitionally compact for every nonempty set $\Theta$ of forward transitions on $(E, D, q)$.

Assuming transitional compactness, Euler method then provides the existence of solutions. It is an important feature of this concept that all existence results can be extended to systems directly.

Proposition 3.14 (Existence of right-hand forward solutions) Assume that $\left(E, q, \Theta^{\mapsto}(E, D, q)\right)$ is transitionally compact. Let $f: E \times[0, T] \longrightarrow \Theta^{\mapsto}(E, D, q)$ fulfill

1. $M:=\sup _{t, y} \alpha^{\mapsto}(f(y, t))<\infty$,

2. $\quad c:=\sup _{t, y} \beta(f(y, t))<\infty$,

3. $\exists \omega(\cdot): Q^{\mapsto}\left(f\left(y_{1}, t_{1}\right), f\left(y_{2}, t_{2}\right)\right) \leq \omega\left(q\left(y_{1}, y_{2}\right)+t_{2}-t_{1}\right) \quad$ for any $0 \leq t_{1} \leq t_{2} \leq T, y_{1}, y_{2} \in E$ and $\omega(h) \searrow 0$ for $h \downarrow 0$.

Then for every initial element $x_{0} \in E$, there is a right-hand forward solution $x:[0, T[\longrightarrow E$ of the generalized mutational equation $\stackrel{\circ}{x}(\cdot) \ni f(x(\cdot), \cdot)$ with $x(0)=x_{0}$.

Proof is based on Euler method for an approximating sequence $\left(x_{n}(\cdot)\right)$ and Cantor diagonal construction for its limit $x(\cdot)$. For $n \in \mathbb{N}\left(2^{n}>T\right)$ set

$$
\begin{array}{rlrl}
h_{n} & :=\frac{T}{2^{n}}, & t_{n}^{j} \quad:=j h_{n} & \text { for } j=0 \ldots 2^{n}, \\
x_{n}(0):=x_{0}, & x_{0}(\cdot):=x_{0}, & \\
x_{n}(t) & :=f\left(x_{n}\left(t_{n}^{j}\right), t_{n}^{j}\right)\left(t-t_{n}^{j}, x_{n}\left(t_{n}^{j}\right)\right) & \text { for } \left.t \in] t_{n}^{j}, t_{n}^{j+1}\right], j \leq 2^{n} . \\
\text { In particular, all } x_{n}(\cdot) \text { satisfy } & q\left(x_{n}(s), x_{n}(t)\right) \leq c \cdot(t-s) & \text { for any } 0 \leq s<t<T+h_{n} .
\end{array}
$$


Now for every $t \in] 0, T\left[\right.$, choose a decreasing sequence $\left(\delta_{k}(t)\right)_{k \in \mathbb{N}}$ in $\mathbb{Q} \cdot T$ satisfying

$$
0<\delta_{k}(t)<\frac{h_{k}}{2}, \quad t+\delta_{k}(t)<T, \quad c \cdot \delta_{k}(t)<h_{k} .
$$

Then, $q\left(x_{n}(t), x_{n}\left(t+\delta_{k}(t)\right)\right) \leq h_{k} \longrightarrow 0$ for $k \longrightarrow \infty$ uniformly in $n$. The transitional compactness provides sequences $m_{k} \nearrow \infty, n_{k} \nearrow \infty\left(m_{k} \leq n_{k}\right)$ of indices and an element $x(t) \in E$ satisfying for every $k \in \mathbb{N}$

$$
\wedge \begin{cases}\sup _{l \geq k} q\left(x_{n_{l}}(t), \quad x(t)\right) & \leq \frac{1}{k}, \\ \sup _{l \geq k} q(x(t), & \left.x_{n_{l}}\left(t+\delta_{m_{k}}(t)\right)\right) \leq \frac{1}{k} .\end{cases}
$$

(In particular, each $m_{k}, n_{k}$ may be replaced by larger indices preserving the properties.) For arbitrary $\kappa \in \mathbb{N}$, these sequences $m_{k}, n_{k} \nearrow \infty$ can even be chosen in such a way that the estimates are fulfilled for the finite set of times $\left.t \in Q_{\kappa}:=\right] 0, T\left[\cap \mathbb{N} \cdot h_{\kappa}\right.$ simultaneously.

Now the Cantor diagonal construction (with respect to the index $\kappa$ ) provides subsequences (again denoted by) $m_{k}, n_{k} \nearrow \infty$ such that both $m_{k} \leq n_{k}$ and for every $\kappa \in \mathbb{N}$, all $s, t \in Q_{\kappa}, k \geq \kappa$

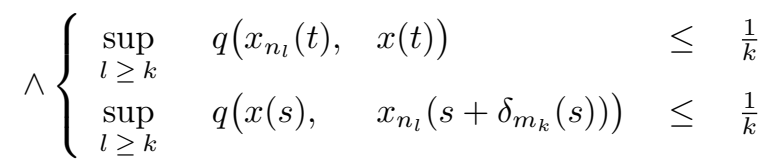

In particular, $q(x(s), x(t)) \leq c \cdot(t-s)$ for any $s, t \in Q_{\mathbb{N}}:=\bigcup_{\kappa} Q_{\kappa}$ with $s<t$. Moreover, the sequence $\left(x_{n_{k}}(\cdot)\right)_{k \in \mathbb{N}}$ fulfills $q\left(x_{n_{k}}(t), x_{n_{l}}\left(t+\delta_{m_{l}}(t)\right)\right) \leq \frac{1}{k}+\frac{1}{l}$ for all $\kappa \in \mathbb{N}, t \in Q_{\kappa}, k, l \geq \kappa$.

For extending $x(\cdot)$ to $t \in] 0, T\left[\backslash Q_{\mathbb{N}}\right.$, we apply the transitional compactness to $\left(\left(x_{n_{k}}(t)\right)_{k \in \mathbb{N}}\right.$ and obtain a subsequence $n_{l_{j}} \nearrow \infty$ of indices (depending on $t$ ) and some $x(t) \in E$ satisfying

$$
\wedge\left\{\begin{array}{lll}
q\left(x_{n_{l_{j}}}(t),\right. & x(t)) & \longrightarrow 0,
\end{array} \quad \text { for } j \longrightarrow \infty\right.
$$

This implies the following convergence even uniformly in $t$

$$
\wedge\left\{\begin{array}{ccc}
\limsup _{\kappa \longrightarrow \infty} & \sup _{k>\kappa} & q\left(x_{n_{k}}\left(t-2 h_{\kappa}\right), \quad x(t)\right)=0, \\
\limsup _{\kappa \longrightarrow \infty} & \sup _{k>\kappa} & q\left(x(t), \quad x_{n_{k}}\left(t+2 h_{\kappa}\right)\right)=0 .
\end{array}\right.
$$

Indeed, for $\kappa \in \mathbb{N}$ fixed arbitrarily and any $t \in] 0, T\left[\right.$, there exists $s=s(t, \kappa) \in Q_{\kappa}$ with

$$
t-2 h_{\kappa}<s \leq t-h_{\kappa} \quad \text { and } \quad q\left(x_{n_{k}}(s), x_{n_{l}}\left(s+\delta_{m_{l}}(s)\right)\right) \leq \frac{1}{k}+\frac{1}{l} \quad \text { for all } k, l \geq \kappa .
$$

So for any $k, l_{j} \geq \kappa$, we conclude from $\delta_{m_{l_{j}}}(\cdot)<\frac{1}{2} h_{m_{l_{j}}} \leq \frac{1}{2} h_{l_{j}} \leq \frac{1}{2} h_{\kappa}$

$$
\begin{array}{rll}
q\left(x_{n_{k}}\left(t-2 h_{\kappa}\right), x(t)\right) \leq & q\left(x_{n_{k}}\left(t-2 h_{\kappa}\right),\right. & \left.x_{n_{k}}(s)\right) \\
& +q\left(x_{n_{k}}(s),\right. & \left.x_{n_{l_{j}}}\left(s+\delta_{m_{l_{j}}}(s)\right)\right) \\
& +q\left(x_{n_{l_{j}}}\left(s+\delta_{m_{l_{j}}}(s)\right),\right. & \left.x_{n_{l_{j}}}(t)\right) \\
& +q\left(x_{n_{l_{j}}}(t),\right. & x(t)) \\
\leq \quad & c \cdot h_{\kappa}+\frac{1}{k}+\frac{1}{l_{j}}+c \cdot 2 h_{\kappa}+q\left(x_{n_{l_{j}}}(t), x(t)\right)
\end{array}
$$

and $j \longrightarrow \infty$ leads to the estimate $q\left(x_{n_{k}}\left(t-2 h_{\kappa}\right), x(t)\right) \leq 2 c \cdot 2 h_{\kappa}+\frac{2}{\kappa}$.

The proof of $\quad \limsup _{\kappa \longrightarrow \infty} \sup _{k>\kappa} q\left(x(t), x_{n_{k}}\left(t+2 h_{\kappa}\right)\right)=0 \quad$ is analogous.

We reformulate the convergence property $(*)$ in the following notation: For each $j \in \mathbb{N}$, there exists some $K_{j} \in \mathbb{N}$ satisfying $K_{j}>K_{j-1}$ and for all $s, t \in\left[0, T\left[, k \geq \kappa \geq K_{j}\right.\right.$,

$$
\wedge\left\{\begin{array}{l}
q\left(x_{n_{k}}\left(s-2 h_{\kappa}\right), \quad x(s)\right) \leq \frac{1}{j} \\
q\left(x(t), \quad x_{n_{k}}\left(t+2 h_{\kappa}\right)\right) \leq \frac{1}{j}
\end{array}\right.
$$


Subsequent Convergence Theorem 3.16 implies that $x(\cdot)$ is a right-hand forward solution of the generalized mutational equation $\stackrel{\circ}{x}(\cdot) \ni f(x, \cdot)$.

Indeed, set $N_{j}:=n_{K_{j}}$ as an abbreviation. Define $g_{j}:(y, t) \longmapsto f\left(x_{N_{j}}\left(t_{N_{j}}^{a+2}+2 h_{K_{j}}\right), t_{N_{j}}^{a+2}+2 h_{K_{j}}\right)$ for $t_{N_{j}}^{a} \leq t<t_{N_{j}}^{a+1}$ and consider the sequence $t \longmapsto x_{N_{j}}\left(t+2 h_{N_{j}}+2 h_{K_{j}}\right)$ of solutions.

Obviously conditions (1.), (3.), (4.) of Proposition 3.16 result from the hypotheses here. Furthermore, we obtain for any $0 \leq t<t^{\prime}<T$ (with $t_{N_{j}}^{a} \leq t<t_{N_{j}}^{a+1}, t_{N_{j}}^{b} \leq t^{\prime}<t_{N_{j}}^{b+1}$ ) and $j \in \mathbb{N}$

$$
\begin{aligned}
& Q^{\mapsto}\left(g_{j}(y, t), \quad g_{j}\left(y^{\prime}, t^{\prime}\right)\right) \\
& =Q^{\mapsto}\left(f\left(x_{N_{j}}\left(t_{N_{j}}^{a+2}+2 h_{K_{j}}\right), t_{N_{j}}^{a+2}+2 h_{K_{j}}\right), f\left(x_{N_{j}}\left(t_{N_{j}}^{b+2}+2 h_{K_{j}}\right), t_{N_{j}}^{b+2}+2 h_{K_{j}}\right)\right) \\
& \leq \widehat{\omega}\left(q\left(x_{N_{j}}\left(t_{N_{j}}^{a+2}+2 h_{K_{j}}\right), x_{N_{j}}\left(t_{N_{j}}^{b+2}+2 h_{K_{j}}\right)\right)+(b-a) h_{N_{j}}\right) \\
& \leq \widehat{\omega}\left(c \cdot\left(t^{\prime}-t+2 h_{N_{j}}\right) \quad+t^{\prime}-t+2 h_{N_{j}}\right) \\
& \longrightarrow 0 \\
& \text { for } j \longrightarrow \infty, t^{\prime}-t \downarrow 0 \text { and all } y, y^{\prime} \text {, }
\end{aligned}
$$

i.e. condition (2.) of Proposition 3.16 is also satisfied by $\left(g_{j}\right)_{j \in \mathbb{N}}$.

Finally for verifying assumption (5.) of Convergence Theorem, we benefit from the convergence properties of $\left(x_{N_{j}}\right)_{j \in \mathbb{N}}$ mentioned before. It ensures that for every $t \in\left[0, T\left[\left(\right.\right.\right.$ with $\left.t_{N_{j}}^{a} \leq t<t_{N_{j}}^{a+1}\right)$,

$$
\begin{aligned}
Q^{\mapsto}\left(f(x(t), t), g_{j}(x(t), t)\right) & =Q^{\mapsto}\left(f(x(t), t), f\left(x_{N_{j}}\left(t_{N_{j}}^{a+2}+2 h_{K_{j}}\right), t_{N_{j}}^{a+2}+2 h_{K_{j}}\right)\right) \\
& \leq \widehat{\omega}\left(q\left(x(t), x_{N_{j}}\left(t_{N_{j}}^{a+2}+2 h_{K_{j}}\right)\right)\right) \\
& \left.\leq \widehat{\omega}\left(q\left(x(t), x_{N_{j}}\left(t+2 h_{K_{j}}\right)\right)\right)+c \cdot 2 h_{K_{j}}+t_{N_{j}}^{a+2}-t\right) \\
& \longrightarrow 0
\end{aligned}
$$

Remark 3.15 1. Assumption (2.) is only to guarantee the uniform continuity of the Euler approximations. If this property results from other arguments, then we can dispense with this assumption and even with condition (4.) of Definition 3.5.

2. The proof in detail shows that the compactness assumption can be weakened slightly.

Considering the initial value problem for $(E, D, q)$, we only need that all values of Euler approximations (at positive times) are contained in a subset $F$ that is transitionally compact in $\left(E, q, \Theta^{\mapsto}(E, D, q)\right)$. In particular, it does not require any additional assumptions about the initial value.

Proposition 3.16 (Convergence Theorem) Suppose the following properties of

$$
\begin{array}{llrlll}
f_{m}, & f: & E \times[0, T[ & \longrightarrow & \Theta^{\mapsto}(E, D, q) & (m \in \mathbb{N}) \\
x_{m}, & x: & {[0, T[} & \longrightarrow & E:
\end{array}
$$

1. $M:=\sup _{m, t, y} \alpha^{\mapsto}\left(f_{m}(y, t)\right)<\infty$,

2. $\limsup Q^{\mapsto}\left(f_{m}\left(y_{1}, t_{1}\right), f_{m}\left(y_{2}, t_{2}\right)\right)=0 \quad$ for $m \longrightarrow \infty, \quad t_{2}-t_{1} \downarrow 0, \quad q\left(y_{1}, y_{2}\right) \longrightarrow 0$,

3. $\stackrel{\circ}{x}_{m}(\cdot) \ni f_{m}\left(x_{m}(\cdot), \cdot\right)$ in $[0, T[$,

4. all $x_{m}(\cdot)$ have a common modulus of continuity $\widehat{\omega}(\cdot)$ with respect to $q$,

5. $\forall t_{1}, t_{2} \in\left[0, T\left[, \quad t_{3} \in\right] 0, T\left[\quad \exists\left(m_{j}\right)_{j \in \mathbb{N}}\right.\right.$ with $m_{j} \nearrow \infty$ and

(i) $\quad \limsup _{j \longrightarrow \infty} Q^{\mapsto}\left(f\left(x\left(t_{1}\right), t_{1}\right), f_{m_{j}}\left(x\left(t_{1}\right), t_{1}\right)\right)=0$,

(ii) $\exists\left(\delta_{j}^{\prime}\right)_{j \in \mathbb{N}}: \delta_{j}^{\prime} \searrow 0, \quad q\left(x\left(t_{2}\right), x_{m_{j}}\left(t_{2}+\delta_{j}^{\prime}\right)\right) \longrightarrow 0$,

(iii) $\exists\left(\delta_{j}\right)_{j \in \mathbb{N}}: \delta_{j} \searrow 0, \quad q\left(x_{m_{j}}\left(t_{3}-\delta_{j}\right), x\left(t_{3}\right)\right) \longrightarrow 0$,

Then, $x(\cdot)$ is a right-hand forward solution of $\stackrel{\circ}{x}(\cdot) \ni f(x(\cdot), \cdot)$ in $[0, T[$. 
Proof. Due to assumption (4.) and (5.), $\widehat{\omega}(\cdot)$ is also the modulus of continuity of $x(\cdot)$.

Now let $z \in D$ and $t \in\left[0, T\left[, \quad 0<h<\mathcal{T}_{\Theta}(f(x(t), t), z)\right.\right.$ be chosen arbitrarily. Condition (6.) of Definition 3.5 ensures for all $k \in] 0, h[$ sufficiently small

$$
q(f(x(t), t)(h, z), x(t+h)) \leq q(f(x(t), t)(h-k, z), x(t+h))+h^{2} .
$$

According to assumptions $(5 . \mathrm{i})-(5 . \mathrm{iii})$, there exist sequences $\left(m_{j}\right)_{j \in \mathbb{N}}, \quad\left(\delta_{j}\right)_{j \in \mathbb{N}}, \quad\left(\delta_{j}^{\prime}\right)_{j \in \mathbb{N}}$ satisfying $m_{j} \nearrow \infty, \quad \delta_{j} \downarrow 0, \quad \delta_{j}^{\prime} \downarrow 0, \quad \delta_{j}+\delta_{j}^{\prime}<k \quad$ and $\quad Q^{\mapsto}\left(f(x(t), t), f_{m_{j}}(x(t), t)\right) \leq h^{2}$,

$$
q\left(x_{m_{j}}\left(t+h-\delta_{j}\right), x(t+h)\right) \longrightarrow 0, \quad q\left(x(t), x_{m_{j}}\left(t+\delta_{j}^{\prime}\right)\right) \longrightarrow 0 .
$$

Thus, subsequent Lemma 3.17 implies for all large $j \in \mathbb{N}$ (depending on $z, t, h, k$ ),

$$
\begin{aligned}
& q(f(x(t), t)(h, z), \quad x(t+h)) \\
\leq & q\left(f(x(t), t)(h-k, z), \quad x_{m_{j}}\left(t+\delta_{j}^{\prime}+h-k\right)\right) \\
& +\quad q\left(x_{m_{j}}\left(t+\delta_{j}^{\prime}+h-k\right), \quad x_{m_{j}}\left(t+h-\delta_{j}\right)\right)
\end{aligned}
$$

Now $j \longrightarrow \infty$ and then $k \longrightarrow 0$ provide the estimate

$q(f(x(t), t)(h, z), x(t+h))$

$\leq q(z, x(t)) \cdot e^{M h}+$ const $\cdot h^{2}+h e^{M h} \cdot \limsup _{j \longrightarrow \infty} \sup _{0 \leq s \leq h} Q^{\mapsto}\left(f_{m_{j}}(x(t), t),\left.f_{m_{j}}\left(x_{m_{j}}, \cdot\right)\right|_{t+\delta_{j}^{\prime}+s}\right)$.

Finally convergence assumption (2.) together with the equi-continuity of $\left(x_{m}(\cdot)\right)_{m \in \mathbb{N}}$ ensures

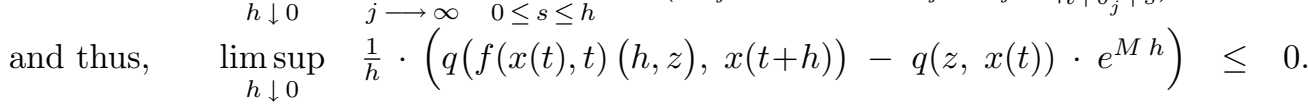

Lemma 3.17 Suppose $\psi \in \Theta^{\mapsto}(E, D, q), \quad z \in D, \quad t_{1} \in\left[0,1\left[, \quad t_{2} \in[0, T[\right.\right.$. Let the curve $x(\cdot):\left[0, T\left[\longrightarrow\right.\right.$ be a right-hand forward primitive of $\vartheta(\cdot):\left[0, T\left[\longrightarrow \Theta^{\mapsto}(E, D, q)\right.\right.$ such that

$$
\wedge\left\{\begin{aligned}
\widehat{\alpha}(\cdot, x, \vartheta) & \leq M(\cdot) \\
Q^{\mapsto}(\psi, \vartheta(\cdot)) & \leq c(\cdot)
\end{aligned}\right.
$$

with upper semicontinuous $M, R, c:\left[0, T\left[\longrightarrow\left[0, \infty\left[\right.\right.\right.\right.$. Set $\mu(h):=\int_{t_{2}}^{t_{2}+h} M(s) d s$.

Then, for every $h \in] 0, T\left[\right.$ with $t_{1}+h<\mathcal{T}_{\Theta}(\psi, z)$,

$$
q\left(\psi\left(t_{1}+h, z\right), x\left(t_{2}+h\right)\right) \leq q\left(\psi\left(t_{1}, z\right), x\left(t_{2}\right)\right) \cdot e^{\mu(h)}+\int_{0}^{h} e^{\mu(h)-\mu(s)} c\left(t_{2}+s\right) d s .
$$

Proof follows essentially the same track as Proposition 3.7 - considering now the auxiliary function $\varphi: h \longmapsto q\left(\psi\left(t_{1}+h, z\right), x\left(t_{2}+h\right)\right)$. Thus, it is not presented here in detail.

Finally, we are interested in bounds of the distance between solutions. However, estimating the distance between points of forward transitions is available only for elements of $D$ in the first argument of $q$ (as in Proposition 3.7). So we use an auxiliary function instead of the distance. Similarly to ordinary differential inclusion, a Lipschitz condition on the right-hand side $f$ comes into play. Furthermore, lower bounds of the time parameter $\mathcal{T}_{\Theta}(\cdot, \cdot)>0$ are assumed for the first time. 
Proposition $3.18 \quad$ Assume for $f: E \times[0, T] \longrightarrow \Theta^{\mapsto}(E, D, q)$ and $x, y:[0, T[\longrightarrow E$

1. $\stackrel{\circ}{x}(\cdot) \ni f(x(\cdot), \cdot), \stackrel{\circ}{y}(\cdot) \ni f(y(\cdot), \cdot) \quad$ in $[0, T[$,

2. $M:=\sup _{t, v} \alpha^{\mapsto}(f(v, t))<\infty$,

3. $\exists \widehat{\omega}(\cdot), L: \quad Q^{\mapsto}\left(f\left(v_{1}, t_{1}\right), f\left(v_{2}, t_{2}\right)\right) \leq L \cdot q\left(v_{1}, v_{2}\right)+\widehat{\omega}\left(t_{2}-t_{1}\right)$

for all $0 \leq t_{1} \leq t_{2} \leq T, \quad v_{1}, v_{2} \in E$ and, $\omega(h) \searrow 0$ for $h \downarrow 0$.

Furthermore suppose for each $t \in\left[0, T\left[\right.\right.$ that the infimum $\varphi(t):=\inf _{z \in D}(q(z, x(t))+q(z, y(t)))<\infty$ can be approximated by a minimizing sequence $\left(z_{j}\right)_{j \in \mathbb{N}}$ in $D$ satisfying $\frac{\sup _{k}>p_{j}\left(z_{j}, z_{k}\right)}{\mathcal{T}_{\Theta}\left(f\left(z_{j}, t\right), z_{j}\right)} \stackrel{j \rightarrow \infty}{\longrightarrow} 0$. Then, $\quad \varphi(t) \leq \varphi(0) e^{(L+M) \cdot t} \quad$ for every time $t \in[0, T[$.

Remark 3.19 1. In the case of symmetric $q$ and $D$ dense in $(E, q)$, we obtain $\varphi(t)=q(x(t), y(t))$.

2. Proving the last proposition, the basic idea consists in estimating both

$$
h \longmapsto q\left(f\left(z_{m}, t\right)\left(h, z_{m}\right), x(t+h)\right) \quad \text { and } \quad h \longmapsto q\left(f\left(z_{m}, t\right)\left(h, z_{m}\right), y(t+h)\right)
$$

(for small $h>0$ ) with such a minimizing sequence $\left(z_{m}\right)_{m \in \mathbb{N}}$ for proving

$$
\liminf _{h \downarrow 0} \frac{\psi(t+h)-\psi(t)}{h} \leq(L+M) \cdot \psi(t) .
$$

Roughly speaking, we need lower bounds of $\mathcal{T}_{\Theta}\left(f\left(z_{m}, t\right), z_{m}\right)$ here for "preserving" the information while $m \longrightarrow \infty$. Finally, the second Lemma of Gronwall (Corollary 3.4) is applied to $\varphi(\cdot)$. We dispense with the proof in detail since this estimate will not be used in $\S 4$. (It is presented completely in [39, 43].)

\section{Nonlocal evolution of compact subsets of $\mathbb{R}^{N}$}

$\mathcal{K}\left(\mathbb{R}^{N}\right)$ consists of all nonempty compact subsets of $\mathbb{R}^{N}$. The so-called Pompeiu-Hausdorff excess is a first example of an ostensible metric on $\mathcal{K}\left(\mathbb{R}^{N}\right)$ that is very similar to the Pompeiu-Hausdorff distance $d$, but not symmetric :

$$
\begin{array}{lll}
\mathbb{e}^{\complement}\left(K_{1}, K_{2}\right) & :=\sup _{x \in K_{1}} & \operatorname{dist}\left(x, K_{2}\right) \\
\mathbb{e}^{\supset}\left(K_{1}, K_{2}\right) & :=\sup _{y \in K_{2}} & \operatorname{dist}\left(y, K_{1}\right) .
\end{array}
$$

for $K_{1}, K_{2} \in \mathcal{K}\left(\mathbb{R}^{N}\right)$. Obviously, the link to the Pompeiu-Hausdorff distance is

$$
d\left(K_{1}, K_{2}\right)=\max \left\{\mathbb{e}^{\subset}\left(K_{1}, K_{2}\right), \mathbb{e}^{\supset}\left(K_{1}, K_{2}\right)\right\}
$$

(see [2, Aubin 99: $\S 3.2]$ and [49, Rockafellar, Wets 98: $\S$ 4.C], for example).

In fact, reachable sets of autonomous differential inclusions provide an example of forward transitions on $\left(\mathcal{K}\left(\mathbb{R}^{N}\right), \mathcal{K}\left(\mathbb{R}^{N}\right), \mathbb{e}^{\supset}\right)$. The well-known Theorem of Filippov (as stated in [5, Aubin 91: Theorem 5.3.1] or [54, Vinter 2000: Theorem 2.4.3]) forms the analytical basis - exactly as in Aubin's original presentation using the Pompeiu-Hausdorff distance $d l[2,3]$. As this example is rather straightforward, we dispense with the details here (see [43, $\S 4.4 .1]$ ).

In the following, we prefer taking the boundaries into consideration explicitly. The Pompeiu-Hausdorff excess $\mathbb{e}^{\supset}\left(K_{1}, K_{2}\right)$, however, does not distinguish between boundary points and interior points of the compact sets $K_{1}, K_{2}$. Thus, an new ostensible metric $q_{\mathcal{K}, N}$ on $\mathcal{K}\left(\mathbb{R}^{N}\right)$ is defined in a moment. Strictly speaking, we even use the first-order approximation of the boundary represented by the limiting normal cones of a set. Following the well-known definitions as in [54, Vinter 2000], these cones are specified:

Notation. Set $\quad \mathbb{B}_{r}(K):=\left\{x \in \mathbb{R}^{N} \mid \operatorname{dist}(x, K) \leq r\right\} \quad$ for any $K \in \mathcal{K}\left(\mathbb{R}^{N}\right)$ and radius $r \geq 0$. As further abbreviations, define $\quad \mathbb{B}_{r}:=\mathbb{B}_{r}(0), \quad \mathbb{B}:=\mathbb{B}_{1}(0) \subset \mathbb{R}^{N}, \quad\|K\|_{\infty}:=\sup _{z \in K}|z|$. 
Definition 4.1 Let $C \subset \mathbb{R}^{N}$ be a nonempty closed set.

$A$ vector $\eta \in \mathbb{R}^{N}, \eta \neq 0$, is said to be a proximal normal vector to $C$ at $x \in C$ if there exists $\rho>0$ with $\mathbb{B}_{\rho}\left(x+\rho \frac{\eta}{|\eta|}\right) \cap C=\{x\}$.

The supremum of all $\rho$ with this property is called proximal radius of $C$ at $x$ in direction $\eta$. The cone of all these proximal normal vectors is called the proximal normal cone to $C$ at $x$ and is abbreviated as $N_{C}^{P}(x)$.

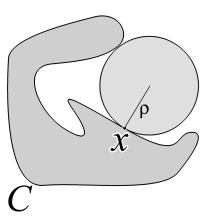

The so-called limiting normal cone $N_{C}(x)$ to $C$ at $x$ consists of all vectors $\eta \in \mathbb{R}^{N}$ that can be approximated by sequences $\left(\eta_{n}\right)_{n \in \mathbb{N}},\left(x_{n}\right)_{n \in \mathbb{N}}$ satisfying

$$
\begin{aligned}
& x_{n} \longrightarrow x, \quad x_{n} \in C, \\
& \eta_{n} \longrightarrow \eta,
\end{aligned}
$$

i.e. $\quad N_{C}(x) \stackrel{\text { Def. }}{=} \operatorname{Limsup} \underset{y \in C}{\underset{y \in C}{\longrightarrow}} N_{C}^{P}(y) \quad$ (in the sense of Painlevé-Kuratowski).

As a further abbreviation, we set ${ }^{b} N_{C}(x):=N_{C}(x) \cap \mathbb{B}=\left\{v \in N_{C}(x):|v| \leq 1\right\}$.

Convention. In the following we restrict ourselves to normal directions at boundary points, i.e. strictly speaking, Graph $N_{C}$ and Graph ${ }^{b} N_{C}$ are the abbreviations of Graph $\left.N_{C}\right|_{\partial C}$, Graph $\left.{ }^{b} N_{C}\right|_{\partial C}$, respectively.

Definition 4.2 Set $q_{\mathcal{K}, N}: \mathcal{K}\left(\mathbb{R}^{N}\right) \times \mathcal{K}\left(\mathbb{R}^{N}\right) \longrightarrow[0, \infty[$,

$$
q_{\mathcal{K}, N}\left(K_{1}, K_{2}\right):=d\left(K_{1}, K_{2}\right)+\mathbb{e}^{\supset}\left(\text { Graph }{ }^{b} N_{K_{1}} \text {, Graph }{ }^{b} N_{K_{2}}\right) .
$$

Obviously, the function $q_{\mathcal{K}, N}$ is a quasi-metric on the set $\mathcal{K}\left(\mathbb{R}^{N}\right)$ of all nonempty compact subsets of $\mathbb{R}^{N}$, i.e. it is positive definite and satisfies the triangle inequality. The properties of $q_{\mathcal{K}, N}$ with respect to convergence depend on the relation between the normal cones of compact sets $K_{n}(n \in \mathbb{N})$ and their limit $K=\operatorname{Lim}_{n \rightarrow \infty} K_{n}$ (if it exists). In general, they do not coincide of course, but each limiting normal vector of $K$ can be approximated by limiting normal vectors of a subsequence $\left(K_{n_{j}}\right)_{j \in \mathbb{N}}$. Stating this inclusion in the next proposition, we regard it as well-known (see e.g. [5, Aubin 91: Theorem 8.4.6] or [22, Cornet, Czarnecki 99: Lemma 4.1]). As the inclusion might be strict, the tuple $\left(\mathcal{K}\left(\mathbb{R}^{N}\right), q_{\mathcal{K}, N}\right)$ is not two-sided compact in the sense of Definition 3.11 .

Proposition 4.3 Let $\left(M_{k}\right)_{k \in \mathbb{N}}$ be a sequence of closed subsets of $\mathbb{R}^{N}$ and set $M:=\operatorname{Limsup}_{k \rightarrow \infty} M_{k}$. Then, 1. Graph $N_{M}^{P} \subset$ Limsup $_{k \rightarrow \infty}$ Graph $N_{M_{k}}^{P}$,

2. Graph $N_{M} \subset \operatorname{Limsup}_{k \rightarrow \infty}$ Graph $N_{M_{k}}$.

Corollary 4.4 Let $\left(M_{k}\right)_{k \in \mathbb{N}}$ be a sequence of closed subsets of $\mathbb{R}^{N}$ whose limit $M:=\operatorname{Lim}_{k \rightarrow \infty} M_{k}$ exists. Then Graph $N_{M} \subset \quad \operatorname{Liminf}_{k \rightarrow \infty}$ Graph $N_{M_{k}}$.

In particular, $\quad \partial M \subset \operatorname{Liminf}_{k \rightarrow \infty} \partial M_{k}$.

Proof is an indirect consequence of Proposition 4.3 due to $M=\operatorname{Lim}_{k \rightarrow \infty} M_{k}$.

Now we focus on the evolution of limiting normal cones at the topological boundary and use the Hamilton condition as a key tool. It implies that roughly speaking, every boundary point $x_{0}$ of $\vartheta_{F}\left(t_{0}, K\right)$ and normal vector $\nu \in N_{\vartheta_{F}\left(t_{0}, K\right)}\left(x_{0}\right)$ have a trajectory and an adjoint arc linking $x_{0}$ to some $z \in \partial K$ and $\nu$ to $N_{K}(z)$, respectively.

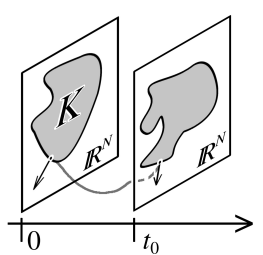




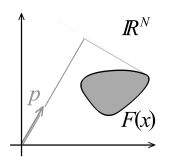

Furthermore the trajectory and its adjoint arc fulfill a system of partial differential equations with the so-called Hamiltonian function of $F: \mathbb{R}^{N} \leadsto \mathbb{R}^{N}$,

$$
\mathcal{H}_{F}: \mathbb{R}^{N} \times \mathbb{R}^{N} \longrightarrow \mathbb{R}^{N}, \quad(x, p) \longmapsto \sup _{y \in F(x)} p \cdot y
$$

Although the Hamilton condition is known in much more general forms (consider, for example, [54, Theorem 7.7.1] applied to proximal balls), we use only the following "smooth" version — due to later regularity conditions on $F$.

\section{Proposition 4.5 Suppose for the set-valued map $F: \mathbb{R}^{N} \leadsto \mathbb{R}^{N}$}

1. $F(\cdot)$ has nonempty convex compact values,

2. $\mathcal{H}_{F}(\cdot, \cdot)$ is continuously differentiable on $\mathbb{R}^{N} \times\left(\mathbb{R}^{N} \backslash\{0\}\right)$,

3. the derivative of $\mathcal{H}_{F}$ has linear growth on $\mathbb{R}^{N} \times\left(\mathbb{R}^{N} \backslash \mathbb{B}_{1}\right)$, i.e.

$\left\|D \mathcal{H}_{F}(x, p)\right\| \leq$ const $\cdot(1+|x|+|p|) \quad$ for all $x, p \in \mathbb{R}^{N},|p|>1$.

Let $K \in \mathcal{K}\left(\mathbb{R}^{N}\right)$ be any initial set and $t_{0}>0$.

For every boundary point $x_{0} \in \partial \vartheta_{F}\left(t_{0}, K\right)$ and normal $\nu \in N_{\vartheta_{F}\left(t_{0}, K\right)}\left(x_{0}\right) \backslash\{0\}$, there exist a solution $x(\cdot) \in C^{1}\left(\left[0, t_{0}\right], \mathbb{R}^{N}\right)$ and its adjoint $p(\cdot) \in C^{1}\left(\left[0, t_{0}\right], \mathbb{R}^{N}\right)$ with

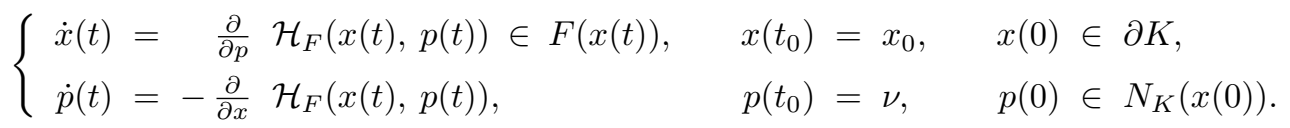

These assumptions give a first hint about adequate conditions on $F: \mathbb{R}^{N} \leadsto \mathbb{R}^{N}$ for inducing forward transitions with respect to $q_{\mathcal{K}, N}$. Supposing $D \mathcal{H}_{F}$ to be Lipschitz continuous (in addition) provides some technical advantages such as global existence of unique solutions of the Hamiltonian system and Remark 4.11 (1.).

Definition 4.6 For $\lambda>0, \quad \operatorname{LIP}_{\lambda}^{(\mathcal{H})}\left(\mathbb{R}^{N}, \mathbb{R}^{N}\right)$ contains all set-valued maps $F: \mathbb{R}^{N} \leadsto \mathbb{R}^{N}$ with

1. $F: \mathbb{R}^{N} \leadsto \mathbb{R}^{N}$ has nonempty compact convex values,

2. $\mathcal{H}_{F}(\cdot, \cdot) \in C^{1,1}\left(\mathbb{R}^{N} \times\left(\mathbb{R}^{N} \backslash\{0\}\right)\right)$,

3. $\left\|\mathcal{H}_{F}\right\|_{C^{1,1}\left(\mathbb{R}^{N} \times \partial \mathbb{B}_{1}\right)} \stackrel{\text { Def. }}{=}\left\|\mathcal{H}_{F}\right\|_{C^{1}\left(\mathbb{R}^{N} \times \partial \mathbb{B}_{1}\right)}+\left.\operatorname{Lip} D \mathcal{H}_{F}\right|_{\mathbb{R}^{N} \times \partial \mathbb{B}_{1}}<\lambda$.

Lemma 4.7 For every $F \in \operatorname{LIP}_{\lambda}^{(\mathcal{H})}\left(\mathbb{R}^{N}, \mathbb{R}^{N}\right)$ and $K \in \mathcal{K}\left(\mathbb{R}^{N}\right), \quad 0 \leq s \leq t \leq T$,

$$
q_{\mathcal{K}, N}\left(\vartheta_{F}(s, K), \vartheta_{F}(t, K)\right) \leq \lambda\left(e^{\lambda T}+2\right) \cdot(t-s) .
$$

Proof. Obviously, the Pompeiu-Hausdorff distance satisfies for every $s, t \geq 0$

$$
d l\left(\vartheta_{F}(s, K), \vartheta_{F}(t, K)\right) \leq \sup _{\mathbb{R}^{N}}\|F(\cdot)\|_{\infty} \cdot(t-s) \leq \lambda(t-s) .
$$

Proposition 4.5 guarantees that for every $0 \leq s<t, x \in \partial \vartheta_{F}(t, K)$ and $p \in{ }^{b} N_{\vartheta_{F}(t, K)}(x) \backslash\{0\}$, there exist a solution $x(\cdot) \in C^{1}\left([s, t], \mathbb{R}^{N}\right)$ and its adjoint $\operatorname{arc} p(\cdot) \in C^{1}\left([s, t], \mathbb{R}^{N}\right)$ satisfying

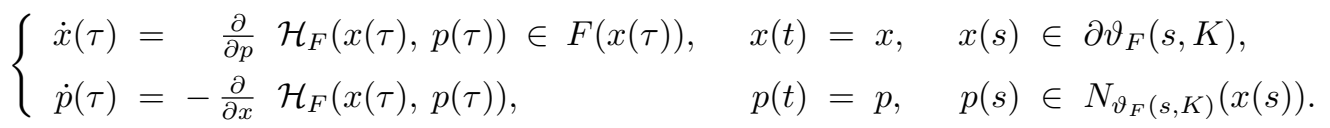

Obviously, $\mathcal{H}_{F}$ is (positively) homogeneous with respect to its second argument and thus, its definition implies $|\dot{p}(\tau)| \leq \lambda|p(\tau)|$ for all $\tau$. Moreover $|p| \leq 1$ implies that the projection of $p$ on any cone is also contained in $\mathbb{B}_{1}$. So finally we obtain 


$$
\begin{aligned}
\operatorname{dist}\left((x, p), \operatorname{Graph}{ }^{b} N_{\vartheta_{F}(s, K)}\right) & \leq|x-x(s)|+|p-p(s)| \\
& \leq\left.\sup _{s \leq \tau \leq t}\left(\left|\frac{\partial}{\partial x} \mathcal{H}_{F}\right|+\left|\frac{\partial}{\partial p} \mathcal{H}_{F}\right|\right)\right|_{(x(\tau), p(\tau))} \cdot(t-s) \\
& \leq \quad\left(\lambda e^{\lambda t}+\lambda\right) \cdot(t-s)
\end{aligned}
$$

So the next question is whether the features of $\operatorname{LIP}_{\lambda}^{(\mathcal{H})}\left(\mathbb{R}^{N}, \mathbb{R}^{N}\right)$ are already sufficient for forward transitions with respect to $q_{\mathcal{K}, N}$. An essential demand is that smooth compact subsets of $\mathbb{R}^{N}$ stay smooth for short times.

Definition 4.8 $\mathcal{K}_{C^{1,1}}\left(\mathbb{R}^{N}\right) \quad$ abbreviates the set of all nonempty compact $N$-dimensional $C^{1,1}$ submanifolds of $\mathbb{R}^{N}$ with boundary.

$A$ closed subset $C \subset \mathbb{R}^{N}$ is said to have positive erosion of radius $\rho>0$ if there exists a closed set $M \subset \mathbb{R}^{N}$ with $C=\left\{x \in \mathbb{R}^{N} \mid \operatorname{dist}(x, M) \leq \rho\right\}$.

$\mathcal{K}_{\circ}^{\rho}\left(\mathbb{R}^{N}\right)$ consists of all sets with positive erosion of radius $\rho>0$ and, set

$$
\mathcal{K}_{\circ}\left(\mathbb{R}^{N}\right):=\bigcup_{\rho>0} \mathcal{K}_{\circ}^{\rho}\left(\mathbb{R}^{N}\right)
$$

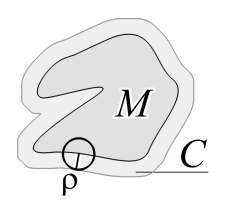

Remark 4.9 The morphological term "erosion" is motivated by the fact that a set $C=\overline{C^{\circ}} \subset \mathbb{R}^{N}$ has positive erosion of radius $\rho>0$ if and only if the closure $\overline{\mathbb{R}^{N} \backslash C}$ of its complement has positive reach in the sense of Federer ([30]).

The relationship between positive reach and positive erosion implies a collection of interesting regularity properties presented (for closed subsets of a Hilbert space) in [21, Clarke, Stern, Wolenski 95], [20, Clarke, Ledyaev, Stern 97], [48, Poliquin, Rockafellar, Thibault 2000].

Proposition 4.10 Let $F: \mathbb{R}^{N} \leadsto \mathbb{R}^{N}$ be a map of $\operatorname{LIP}_{\lambda}^{(\mathcal{H})}\left(\mathbb{R}^{N}, \mathbb{R}^{N}\right)$. For every compact $N$ dimensional $C^{1,1}$ submanifold $K$ of $\mathbb{R}^{N}$ with boundary, there exist a time $\tau>0$ and a radius $\rho>0$ such that for all $t \in[0, \tau[$,

1. $\vartheta_{F}(t, K) \in \mathcal{K}_{C^{1,1}}\left(\mathbb{R}^{N}\right)$ with radius of curvature $\geq \rho$,

(i.e. $\vartheta_{F}(t, K)$ has both positive reach and positive erosion of radius $\geq \rho$ ).

2. $K=\mathbb{R}^{N} \backslash \vartheta_{-F}\left(t, \mathbb{R}^{N} \backslash \vartheta_{F}(t, K)\right)$.

Remark 4.11 1. A complete proof is presented in the appendix (Propositions A.2, A.4). For statement (1.), we use the evolution of Graph $\left(N_{K}(\cdot) \cap \partial \mathbb{B}\right) \subset \mathbb{R}^{N} \times \mathbb{R}^{N}$ along the Hamiltonian system with $\mathcal{H}_{F}$. Indeed, Lemma A.3 specifies sufficient conditions on the system so that graphs of Lipschitz continuous functions preserve this regularity for short times. Applying this lemma to unit normals to reachable sets of $K \in \mathcal{K}_{C^{1,1}}\left(\mathbb{R}^{N}\right)$ requires the Hamiltonian $\mathcal{H}_{F}$ to be in $C^{1,1}\left(\mathbb{R}^{N} \times\left(\mathbb{R}^{N} \backslash\{0\}\right)\right)$ instead of $C^{1}$. In fact, this Lemma A.3 is an analytical reason for choosing $\mathcal{K}_{C^{1,1}}\left(\mathbb{R}^{N}\right)$ as "test subset" of $\mathcal{K}\left(\mathbb{R}^{N}\right)$ - instead of compact sets with $C^{1}$ boundary, for example.

2. Together with Proposition 4.5, statement (2.) provides a connection between the boundaries $\partial K$ and $\partial \vartheta_{F}(t, K)$ - now in both forward and backward time direction. 
Lemma 4.12 Assume for $F, G \in \operatorname{LIP}_{\lambda}^{(\mathcal{H})}\left(\mathbb{R}^{N}, \mathbb{R}^{N}\right), K_{1}, K_{2} \in \mathcal{K}\left(\mathbb{R}^{N}\right)$ and $T>0$ that all the sets $\vartheta_{F}\left(t, K_{1}\right) \in \mathcal{K}_{C^{1,1}}\left(\mathbb{R}^{N}\right) \quad(0 \leq t \leq T)$ have uniform positive reach.

Then, for every $t \in[0, T[$,

$$
\begin{aligned}
& q_{\mathcal{K}, N}\left(\vartheta_{F}\left(t, K_{1}\right), \vartheta_{G}\left(t, K_{2}\right)\right) \leq e^{\left(\Lambda_{F}+\lambda\right) t} \cdot\left(q_{\mathcal{K}, N}\left(K_{1}, K_{2}\right)+6 N t\left\|\mathcal{H}_{F}-\mathcal{H}_{G}\right\|_{C^{1}\left(\mathbb{R}^{N} \times \partial \mathbb{B}_{1}\right)}\right) \\
& \text { with } \quad \Lambda_{F}:=9 e^{2 \lambda T}\left\|\mathcal{H}_{F}\right\|_{C^{1,1}\left(\mathbb{R}^{N} \times \partial \mathbb{B}_{1}\right)} \leq 9 e^{2 \lambda T} \lambda<\infty .
\end{aligned}
$$

Proof. [2, Proposition 3.7.3] concludes the following estimate of the Pompeiu-Hausdorff distance directly from Filippov's well-known Theorem about differential inclusions (with Lipschitz continuous right-hand side)

$$
\begin{aligned}
d l\left(\vartheta_{F}\left(t, K_{1}\right), \vartheta_{G}\left(t, K_{2}\right)\right) & \leq d l\left(K_{1}, K_{2}\right) \cdot e^{\lambda t}+\sup _{\mathbb{R}^{N}} d(F(\cdot), G(\cdot)) \cdot \frac{e^{\lambda t}-1}{\lambda} \\
& \leq d l\left(K_{1}, K_{2}\right) \cdot e^{\lambda t}+\sup _{\mathbb{R}^{N} \times \partial \mathbb{B}_{1}}\left|\mathcal{H}_{F}-\mathcal{H}_{G}\right| \cdot t e^{\lambda t} .
\end{aligned}
$$

So now we need an upper bound of $\mathbb{e}^{\supset}\left(\operatorname{Graph}^{b} N_{\vartheta_{F}\left(t, K_{1}\right)}\right.$, Graph $\left.{ }^{b} N_{\vartheta_{G}\left(t, K_{2}\right)}\right)$.

Choose $x \in \partial \vartheta_{G}\left(t, K_{2}\right), \quad p \in N_{\vartheta_{G}\left(t, K_{2}\right)}(x) \cap \partial \mathbb{B}_{1}$ and $\delta>0$ arbitrarily. According to Proposition 4.5, there exist a solution $x(\cdot) \in C^{1}\left([0, t], \mathbb{R}^{N}\right)$ of $G$ and its adjoint arc $p(\cdot) \in C^{1}\left([0, t], \mathbb{R}^{N}\right)$ with

$$
\begin{array}{rlrl}
\dot{x}(\cdot) & =\frac{\partial}{\partial p} \mathcal{H}_{G}(x(\cdot), p(\cdot)) \in G(x(\cdot)), & \dot{p}(\cdot) & =-\frac{\partial}{\partial x} \mathcal{H}_{G}(x(\cdot), p(\cdot)) \in \lambda|p(\cdot)| \cdot \mathbb{B} \\
x(0) & \in \partial K_{2}, \quad x(t)=x, & p(0) \in N_{K_{2}}(x(0)), \quad p(t)=p .
\end{array}
$$

Gronwall's Lemma guarantees

$$
0<e^{-\lambda t} \leq|p(\cdot)| \leq e^{\lambda t}
$$

and so, $\quad p(0) e^{-\lambda t} \in{ }^{b} N_{K_{2}}(x(0)) \backslash\{0\}$.

Now let $\left(y_{0}, \widehat{q}_{0}\right)$ denote an element of Graph ${ }^{b} N_{K_{1}}$ with $\widehat{q}_{0} \neq 0$ and

$$
\begin{aligned}
& \left|\left(y_{0}, \widehat{q}_{0}\right)-\left(x(0), p(0) e^{-\lambda t}\right)\right| \\
\leq & \mathbb{e}^{\supset}\left(\text { Graph }^{b} N_{K_{1}}, \text { Graph }^{b} N_{K_{2}}\right)+\delta .
\end{aligned}
$$

Assuming that all $\vartheta_{F}\left(s, K_{1}\right) \in \mathcal{K}\left(\mathbb{R}^{N}\right)(s \in[0, t])$ have uniform positive reach implies the reversibility in time due to Prop. A.4: $\mathbb{R}^{N} \backslash K_{1}=\vartheta_{-F}\left(t, \mathbb{R}^{N} \backslash \vartheta_{F}\left(t, K_{1}\right)\right)$.

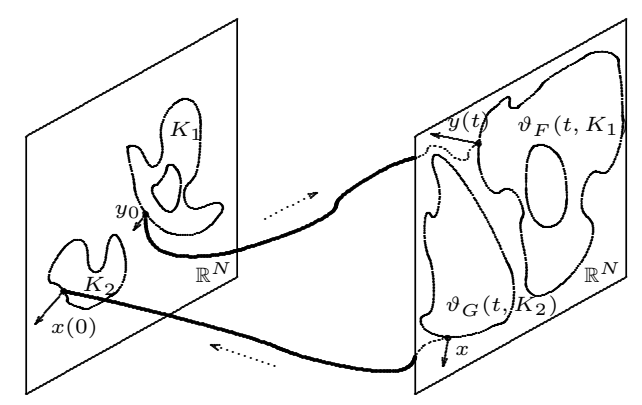

$s=0$ time

So in particular, $y_{0}$ is a boundary point of the (not compact) $N$-dimensional $C^{1,1}$ submanifold $\mathbb{R}^{N} \backslash \stackrel{\circ}{K}_{1}=\vartheta_{-F}\left(t, \overline{\mathbb{R}^{N} \backslash \vartheta_{F}\left(t, K_{1}\right)}\right)$ with boundary and, $-\widehat{q}_{0}$ belongs to its limiting normal cone at $y_{0}$. As a consequence of Prop. 4.5 again and due to $\mathcal{H}_{-F}(z, v)=\mathcal{H}_{F}(z,-v)$ for all $z, v$, we obtain a solution $y(\cdot) \in C^{1}\left([0, t], \mathbb{R}^{N}\right)$ and its adjoint arc $q(\cdot)$ satisfying

$$
\begin{aligned}
\dot{y}(\cdot) & =\frac{\partial}{\partial p} \mathcal{H}_{F}(y(\cdot), q(\cdot)), & \dot{q}(\cdot) & =-\frac{\partial}{\partial y} \mathcal{H}_{F}(y(\cdot), q(\cdot)) \\
y(0) & =y_{0}, & q(0) & =\widehat{q}_{0} e^{\lambda t} \neq 0, \\
y(t) & \in \partial \vartheta_{F}\left(t, K_{1}\right), & q(t) & \in N_{\vartheta_{F}\left(t, K_{1}\right)}(y(t)) .
\end{aligned}
$$

According to Lemma 4.13 , the derivative of $\mathcal{H}_{F}$ is $\Lambda_{F}$-Lipschitz continuous on $\mathbb{R}^{N} \times\left(\mathbb{B}_{e^{\lambda T}} \backslash \stackrel{\circ}{\mathbb{B}_{e^{-\lambda T}}}\right)$. Thus, the Theorem of Cauchy-Lipschitz leads to

$$
\begin{aligned}
& \operatorname{dist}\left((x, p) \text {, Graph }{ }^{b} N_{\vartheta_{F}\left(t, K_{1}\right)}\right) \leq|(x, p)-(y(t), q(t))| \\
& \leq e^{\Lambda_{F} \cdot t} \cdot\left|(x(0), p(0))-\left(y_{0}, \widehat{q}_{0} e^{\lambda t}\right)\right|+\left.\frac{e^{\Lambda_{F} \cdot t}-1}{\Lambda_{F}} \cdot \sup _{0 \leq s \leq t}\left|D \mathcal{H}_{F}-D \mathcal{H}_{G}\right|\right|_{(x(s), p(s))} .
\end{aligned}
$$

$\mathcal{H}_{F}$ and $\mathcal{H}_{G}$ are positively homogenous with respect to the second argument and thus,

$$
\begin{aligned}
&\left|\frac{\partial}{\partial x_{j}}\left(\mathcal{H}_{F}-\mathcal{H}_{G}\right)\right|_{(x(s), p(s))} \mid \leq e^{\lambda t}\left\|D \mathcal{H}_{F}-D \mathcal{H}_{G}\right\|_{C^{0}\left(\mathbb{R}^{N} \times \partial \mathbb{B}_{1}\right)}, \\
&\left|\frac{\partial}{\partial p_{j}}\left(\mathcal{H}_{F}-\mathcal{H}_{G}\right)\right|_{(x(s), p(s))} \mid \leq 3 \cdot\left\|\mathcal{H}_{F}-\mathcal{H}_{G}\right\|_{C^{1}\left(\mathbb{R}^{N} \times \partial \mathbb{B}_{1}\right)} .
\end{aligned}
$$


So we obtain

$\operatorname{dist}\left((x, p)\right.$, Graph $\left.{ }^{b} N_{\vartheta_{F}\left(t, K_{1}\right)}\right)$

$\leq e^{\left(\Lambda_{F}+\lambda\right) t}\left|\left(x(0), p(0) e^{-\lambda t}\right)-\left(y_{0}, \widehat{q}_{0}\right)\right|+e^{\Lambda_{F} t} t \cdot 6 N e^{\lambda t}\left\|\mathcal{H}_{F}-\mathcal{H}_{G}\right\|_{C^{1}\left(\mathbb{R}^{N} \times \partial \mathbb{B}_{1}\right)}$

and, since $\delta>0$ is arbitrarily small and $|p|=1$,

$e^{\supset}\left(\right.$ Graph ${ }^{b} N_{\vartheta_{F}\left(t, K_{1}\right)}$, Graph $\left.{ }^{b} N_{\vartheta_{G}\left(t, K_{2}\right)}\right)$

$\leq e^{\left(\Lambda_{F}+\lambda\right) t} \cdot\left\{e^{\supset}\left(\operatorname{Graph}^{b} N_{K_{1}}\right.\right.$, Graph $\left.\left.^{b} N_{K_{2}}\right)+6 N t \cdot\left\|\mathcal{H}_{F}-\mathcal{H}_{G}\right\|_{C^{1}\left(\mathbb{R}^{N} \times \partial \mathbb{B}_{1}\right)}\right\}$.

Lemma 4.13 For every $F \in \operatorname{LIP}_{\lambda}^{(\mathcal{H})}\left(\mathbb{R}^{N}, \mathbb{R}^{N}\right)$ and radius $R>1$, the product $9 R^{2} \lambda$ is a Lipschitz constant of the derivative $D \mathcal{H}_{F}$ restricted to $\mathbb{R}^{N} \times\left(\mathbb{B}_{R} \backslash \stackrel{\circ}{\mathbb{B}_{\frac{1}{R}}}\right)$.

Proof results from the fact that $\mathcal{H}_{F}(x, p)$ is positively homogenous with respect to $p$. (For further details see [43, Lemma 4.4.24].)

Proposition 4.14 For every $\lambda \geq 0$, the reachable sets of the set-valued maps in $\operatorname{LIP}_{\lambda}^{(\mathcal{H})}\left(\mathbb{R}^{N}, \mathbb{R}^{N}\right)$ induce forward transitions on $\left(\mathcal{K}\left(\mathbb{R}^{N}\right), \mathcal{K}_{C^{1,1}}\left(\mathbb{R}^{N}\right), q_{\mathcal{K}, N}\right)$

with

$$
\begin{array}{rlrll}
\alpha^{\mapsto}\left(\vartheta_{F}\right) & \stackrel{\text { Def. }}{=} & 10 & \lambda & \\
\beta\left(\vartheta_{F}\right) & \stackrel{\text { Def. }}{=} & \lambda & \left(e^{\lambda}+2\right), \\
Q^{\mapsto}\left(\vartheta_{F}, \vartheta_{G}\right) & \leq & 6 & N & \left\|\mathcal{H}_{F}-\mathcal{H}_{G}\right\|_{C^{1}\left(\mathbb{R}^{N} \times \partial \mathbb{B}_{1}\right)} .
\end{array}
$$

Proof. $\quad$ The semigroup property of reachable sets implies again

$$
\begin{aligned}
& q_{\mathcal{K}, N}\left(\vartheta_{F}\left(h, \vartheta_{F}(t, K)\right), \quad \vartheta_{F}(t+h, K)\right)=0, \\
& q_{\mathcal{K}, N}\left(\vartheta_{F}(t+h, K), \quad \vartheta_{F}\left(h, \vartheta_{F}(t, K)\right)\right)=0
\end{aligned}
$$

for all $F \in \operatorname{LIP}_{\lambda}^{(\mathcal{H})}\left(\mathbb{R}^{N}, \mathbb{R}^{N}\right), \quad K \in \mathcal{K}\left(\mathbb{R}^{N}\right), h, t \geq 0$ since $q_{\mathcal{K}, N}$ is a quasi-metric.

According to Proposition 4.10, every set-valued map $F \in \operatorname{LIP}_{\lambda}^{(\mathcal{H})}\left(\mathbb{R}^{N}, \mathbb{R}^{N}\right)$ and initial set $K_{1} \in$ $\mathcal{K}_{C^{1,1}}\left(\mathbb{R}^{N}\right)$ lead to a time $\mathcal{T}_{\Theta}\left(\vartheta_{F}, K_{1}\right)>0$ and a radius $\rho>0$ such that $\vartheta_{F}\left(t, K_{1}\right) \in \mathcal{K}_{C^{1,1}}\left(\mathbb{R}^{N}\right)$ has positive reach of radius $\geq \rho$ for any $t \in\left[0, \mathcal{T}_{\Theta}\left(\vartheta_{F}, K_{1}\right)\right]$.

So Lemma 4.12 guarantees for all $K_{1} \in \mathcal{K}_{C^{1,1}}\left(\mathbb{R}^{N}\right)$ and $K_{2} \in \mathcal{K}\left(\mathbb{R}^{N}\right)$ with $K_{1} \neq K_{2}$

$$
\begin{aligned}
& \limsup _{h \downarrow 0}\left(\frac{q \mathcal{K}, N}{\left(\vartheta_{F}\left(h, K_{1}\right), \vartheta_{F}\left(h, K_{2}\right)\right)-q_{\mathcal{K}, N}\left(K_{1}, K_{2}\right)}\right)^{h} q_{\mathcal{K}, N}\left(K_{1}, K_{2}\right) \\
\leq & \limsup _{h \downarrow 0} \frac{1}{h}\left(e^{\left(9 e^{2 \lambda h} \lambda+\lambda\right) \cdot h}-1\right)=10 \lambda \stackrel{\text { Def. }}{=} \alpha^{\mapsto}\left(\vartheta_{F}\right)
\end{aligned}
$$

and for every $F, G \in \operatorname{LIP}_{\lambda}^{(\mathcal{H})}\left(\mathbb{R}^{N}, \mathbb{R}^{N}\right)$

$$
\begin{aligned}
& Q^{\mapsto}\left(\vartheta_{F}, \vartheta_{G}\right) \leq \sup _{\substack{K_{1} \in \mathcal{K}_{C^{1,1}}\left(\mathbb{R}^{N}\right) \\
K_{2} \in \mathcal{K}\left(\mathbb{R}^{N}\right)}} \limsup _{h \downarrow 0}\left(q_{\mathcal{K}, N}\left(K_{1}, K_{2}\right) \frac{1}{h}\left(e^{\left(9 e^{2 \lambda h} \lambda+\lambda\right) \cdot h}-e^{10 \lambda h}\right)\right. \\
& \left.+6 N \cdot\left\|\mathcal{H}_{F}-\mathcal{H}_{G}\right\|_{C^{1}\left(\mathbb{R}^{N} \times \partial \mathbb{B}_{1}\right)} e^{\left(9 e^{2 \lambda h} \lambda+\lambda\right) \cdot h}\right) \\
& =\quad 6 N \cdot\left\|\mathcal{H}_{F}-\mathcal{H}_{G}\right\|_{C^{1}\left(\mathbb{R}^{N} \times \partial \mathbb{B}_{1}\right)} .
\end{aligned}
$$

Moreover Lemma 4.7 states

$$
q_{\mathcal{K}, N}\left(\vartheta_{F}(s, K), \vartheta_{F}(t, K)\right) \leq \lambda\left(e^{\lambda}+2\right) \cdot(t-s)
$$

for any $0 \leq s \leq t \leq 1$ and $K \in \mathcal{K}\left(\mathbb{R}^{N}\right)$.

Finally we have to show $\quad \limsup _{h \downarrow 0} q_{\mathcal{K}, N}\left(\vartheta_{F}\left(t-h, K_{1}\right), K_{2}\right) \geq q_{\mathcal{K}, N}\left(\vartheta_{F}\left(t, K_{1}\right), K_{2}\right) \quad$ for all $F \in \operatorname{LIP}_{\lambda}^{(\mathcal{H})}\left(\mathbb{R}^{N}, \mathbb{R}^{N}\right), K_{1} \in \mathcal{K}_{C^{1,1}}\left(\mathbb{R}^{N}\right), K_{2} \in \mathcal{K}\left(\mathbb{R}^{N}\right)$ and $0<t<\mathcal{T}_{\Theta}\left(\vartheta_{F}, K_{1}\right)$.

Proposition A.4 ensures the reversibility in time in the interval $\left[0, \mathcal{T}_{\Theta}\left(\vartheta_{F}, K_{1}\right)\right.$, i.e.

$$
\mathbb{R}^{N} \backslash \vartheta_{F}\left(t-h, K_{1}\right)=\vartheta_{-F}\left(h, \mathbb{R}^{N} \backslash \vartheta_{F}\left(t, K_{1}\right)\right) \quad \text { for every } 0<h<t<\mathcal{T}_{\Theta}\left(\vartheta_{F}, K_{1}\right) .
$$


Due to standard hypothesis $(\mathcal{H})$, the flow of the Hamiltonian system even induces a Lipschitz homeomorphism between Graph $N_{\vartheta_{F}\left(t-h, K_{1}\right)}$ and Graph $N_{\vartheta_{F}\left(t, K_{1}\right)}$ since each limiting normal cone contains exactly one direction and $N_{\vartheta_{F}\left(t, K_{1}\right)}(\cdot)=-N_{\overline{\mathbb{R}^{N} \backslash \vartheta_{F}\left(t, K_{1}\right)}}(\cdot)$.

Thus, Graph $N_{\vartheta_{F}\left(t, K_{1}\right)}=\operatorname{Lim}_{h \downarrow 0}$ Graph $N_{\vartheta_{F}\left(t-h, K_{1}\right)}$ and finally,

$$
q_{\mathcal{K}, N}\left(\vartheta_{F}\left(t, K_{1}\right), \vartheta_{F}\left(t-h, K_{1}\right)\right) \quad \longrightarrow 0 \quad \text { for } h \downarrow 0 .
$$

So the last claim results from the triangle inequality.

For applying Proposition 3.14 about the existence of right-hand forward solutions, we still need sufficient conditions for the transitional compactness.

Definition 4.15 For any $\lambda>0$ and $\rho>0$, the set $\operatorname{LIP}_{\lambda}^{\left(\mathcal{H}_{\circ}^{\rho}\right)}\left(\mathbb{R}^{N}, \mathbb{R}^{N}\right)$ consists of all set-valued maps $F: \mathbb{R}^{N} \leadsto \mathbb{R}^{N}$ satisfying

1. $F: \mathbb{R}^{N} \leadsto \mathbb{R}^{N}$ has compact convex values in $\mathcal{K}_{\circ}^{\rho}\left(\mathbb{R}^{N}\right)$.

2. $\mathcal{H}_{F}(\cdot, \cdot) \in C^{2}\left(\mathbb{R}^{N} \times\left(\mathbb{R}^{N} \backslash\{0\}\right)\right)$,

3. $\left\|\mathcal{H}_{F}\right\|_{C^{1,1}\left(\mathbb{R}^{N} \times \partial \mathbb{B}_{1}\right)} \stackrel{\text { Def. }}{=}\left\|\mathcal{H}_{F}\right\|_{C^{1}\left(\mathbb{R}^{N} \times \partial \mathbb{B}_{1}\right)}+\left.\operatorname{Lip} D \mathcal{H}_{F}\right|_{\mathbb{R}^{N} \times \partial \mathbb{B}_{1}}<\lambda$.

Remark 4.16 $\operatorname{LIP}_{\lambda}^{\left(\mathcal{H}_{\circ}^{\rho}\right)}\left(\mathbb{R}^{N}, \mathbb{R}^{N}\right)$ is a subset of $\operatorname{LIP}_{\lambda}^{(\mathcal{H})}\left(\mathbb{R}^{N}, \mathbb{R}^{N}\right)$ and its maps fulfill standard hypothesis $\left(\mathcal{H}_{\circ}^{\rho}\right) \quad$ (see Definition A.7). In particular, they make points evolve into sets of positive erosion according to Proposition A.9.

\section{Proposition 4.17}

For any $\lambda, \rho>0$, consider the maps $F \in \operatorname{LIP}_{\lambda}^{\left(\mathcal{H}_{\circ}^{\rho}\right)}\left(\mathbb{R}^{N}, \mathbb{R}^{N}\right)$ (i.e. their reachable sets, strictly speaking) as forward transitions on $\left(\mathcal{K}\left(\mathbb{R}^{N}\right), \mathcal{K}_{C^{1,1}}\left(\mathbb{R}^{N}\right), q_{\mathcal{K}, N}\right)$.

Then $\mathcal{K}_{\circ}\left(\mathbb{R}^{N}\right)$ is transitionally compact in $\left(\mathcal{K}\left(\mathbb{R}^{N}\right), q_{\mathcal{K}, N}, \operatorname{LIP}_{\lambda}^{\left(\mathcal{H}_{\circ}^{\rho}\right)}\left(\mathbb{R}^{N}, \mathbb{R}^{N}\right)\right) \quad$ in the following sense (see Definitions 3.12, 4.8) : Let $\left(K_{n}\right)_{n \in \mathbb{N}},\left(h_{j}\right)_{j \in \mathbb{N}}$ be sequences in $\mathcal{K}_{\circ}\left(\mathbb{R}^{N}\right)$ and $] 0,1[$, respectively with $h_{j} \downarrow 0, \sup _{n} q_{\mathcal{K}, N}\left(\mathbb{B}_{1}, K_{n}\right)<\infty$. Suppose each $G_{n}:[0,1] \longrightarrow \operatorname{LIP}_{\lambda}^{\left(\mathcal{H}_{\circ}^{\rho}\right)}\left(\mathbb{R}^{N}, \mathbb{R}^{N}\right)$ to be piecewise constant $(n \in \mathbb{N})$ and set

$$
\begin{array}{ll}
\widetilde{G}_{n}:[0,1] \times \mathbb{R}^{N} \leadsto \mathbb{R}^{N},(t, x) \longmapsto & G_{n}(t)(x), \\
K_{n}(h):=\vartheta_{\widetilde{G}_{n}}\left(h, K_{n}\right) & \text { for } h \geq 0 .
\end{array}
$$

Then there exist a sequence $n_{k} \nearrow \infty$ of indices and $K \in \mathcal{K}\left(\mathbb{R}^{N}\right)$ satisfying

$$
\begin{aligned}
\limsup _{k \longrightarrow \infty} q_{\mathcal{K}, N}\left(K_{n_{k}}(0), \quad K\right)=0, \\
\limsup _{j \longrightarrow \infty} \quad \sup _{k \geq j} q_{\mathcal{K}, N}\left(K, K_{n_{k}}\left(h_{j}\right)\right)=0 .
\end{aligned}
$$

Proof. $\quad$ Closed bounded balls in $\left(\mathcal{K}\left(\mathbb{R}^{N}\right), d l\right)$ are known to be compact. So there exist a subsequence (again denoted by) $\left(K_{n}\right)_{n \in \mathbb{N}}$ and $K \in \mathcal{K}\left(\mathbb{R}^{N}\right)$ with $d l\left(K_{n}, K\right) \longrightarrow 0(n \longrightarrow \infty)$. Thus, $\quad d l\left(K, K_{n}(h)\right) \leq d l\left(K, K_{n}\right)+\lambda h \longrightarrow \lambda h$ for $n \longrightarrow \infty$. Furthermore Corollary 4.4 implies $q_{\mathcal{K}, N}\left(K_{n}, K\right) \longrightarrow 0$.

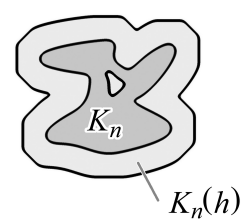

Now we want to prove that $K$ satisfies the claim by choosing subsequences of $\left(K_{n}\right)$ for countably many times and finally applying the Cantor diagonal construction.

An important tool here is Proposition A.9. It ensures the existence of $\sigma=\sigma(\lambda, \rho, K)>0$ and $\widehat{h}=$ $\widehat{h}(\lambda, \rho, K) \in] 0,1]$ such that $\vartheta_{-} \widetilde{G}_{n}(h-\cdot, \cdot)(h, z)$ has positive erosion of radius $\sigma h$ for every $\left.\left.h \in\right] 0, \widehat{h}\right]$ and $z \in \mathbb{B}_{1}(K)$. In the following, we assume without loss of generality $0<h_{j}<\widehat{h}$ and $K_{n}(h) \subset \mathbb{B}_{1}(K)$ for all $j, n \in \mathbb{N}, h \in[0, \widehat{h}]$. 
So the asymptotic properties of have to be investigated for each $h \in] 0, \widehat{h}]$.

$$
\mathbb{e}^{\supset}\left(\text { Graph }{ }^{b} N_{K} \text {, Graph }{ }^{b} N_{K_{n}(h)}\right) \quad(n \longrightarrow \infty)
$$

Due to Definition 4.1, every limiting normal cone results from the neighboring proximal normal cones, i.e. $\quad N_{C}(x) \stackrel{\text { Def. }}{=}$ Limsup $\underset{y \in C}{\rightarrow \rightarrow x} N_{C}^{P}(y) \quad$ for all nonempty $C \subset \mathbb{R}^{N}, x \in \partial C$.

Thus, Graph $N_{C}=\overline{\text { Graph } N_{C}^{P}}$ and from now on, we confine our considerations to the excess $\mathbb{e}^{\supset}\left(\right.$ Graph ${ }^{b} N_{K}$, Graph $\left.{ }^{b} N_{K_{n}(h)}^{P}\right)$ for any $\left.\left.h \in\right] 0, \widehat{h}\right]$.
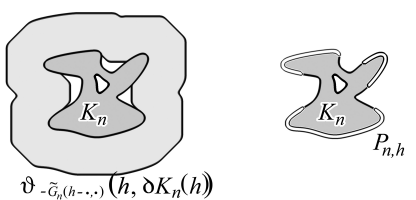

$P_{n, h}:=K_{n} \cap \vartheta_{-\widetilde{G}_{n}(h-\cdot, \cdot)}\left(h, \partial K_{n}(h)\right)$ is a subset of $\partial K_{n}$. More precisely, it consists of all points $x \in K_{n}$ such that a trajectory of $\widetilde{G}_{n}$ starts in $x$ and reaches $\partial K_{n}(h)$ at time $h$. In addition, every boundary point $y$ of $K_{n}(h)$ is attained by such a trajectory.

Taking now adjoint arcs into account, the Hamiltonian system in Proposition 4.5 provides the following estimate for every $n \in \mathbb{N}$ (similarly to Lemma 4.7)

$$
\mathbb{e}^{\supset}\left(\text { Graph }\left.{ }^{b} N_{K_{n}}\right|_{P_{n, h}} \text {, Graph }{ }^{b} N_{K_{n}(h)}^{P}\right) \leq \operatorname{const}(\lambda) \cdot h .
$$

The next step provides the identity of normals: Graph ${ }^{b} N_{K_{n}} \mid P_{n, h}=$ Graph ${ }^{b} N_{K_{n}}^{P} \mid P_{n, h}$.

Indeed, $\quad N_{\mathbb{R}^{N} \backslash K_{n}}^{P}(x) \neq \emptyset \quad$ for all $x \in \partial K_{n}, \quad$ due to $K_{n} \in \mathcal{K}_{\circ}\left(\mathbb{R}^{N}\right)$.

In particular, $N_{K_{n}}^{P}(x) \quad \neq \emptyset$ for all $x \in P_{n, h} \quad$ since $\vartheta_{-\widetilde{G}_{n}(h-\cdot, \cdot)}\left(h, \partial K_{n}(h)\right)$ has positive erosion of radius $\sigma h$ (Proposition A.9) and $K_{n} \cap\left(\vartheta_{-\widetilde{G}_{n}(h-., \cdot)}\left(h, \partial K_{n}(h)\right)\right)^{\circ}=\emptyset$.

So, $\quad N_{\mathbb{R}^{N} \backslash K_{n}}^{P}(x)=-N_{K_{n}}^{P}(x)$ contain exactly one direction for every point $x \in P_{n, h}$ according to [20, Clarke,Ledyaev,Stern 97: Lemma 6.4].

The positive erosion of $K_{n}$ implies that $\overline{\mathbb{R}^{N} \backslash K_{n}}$ has positive reach and thus, $N_{\mathbb{R}^{N} \backslash K_{n}}^{P}(x)=$ $N_{\overline{\mathbb{R}^{N} \backslash K_{n}}}(x)=N_{\overline{\mathbb{R}}^{N} \backslash K_{n}}^{C}(x)$ contain exactly one direction (with $N_{M}^{C}(x)$ denoting the Clarke normal cone of $M \subset \mathbb{R}^{N}$ at $x$ ). Due to a well-known result about Clarke normal cones of closed complements in [19, Clarke 83], we obtain that $N_{K_{n}}^{C}(x)=-N_{\mathbb{R}^{N} \backslash K_{n}}^{C}(x)$ consist of exactly one direction for all $x \in P_{n, h}$ and so,

$$
N_{K_{n}}^{C}(x)=N_{K_{n}}(x)=N_{K_{n}}^{P}(x)
$$

In addition, the proximal radius of $K_{n}$ at each $x \in P_{n, h}$ (in its unique proximal direction) is $\geq \sigma h$ since $\vartheta_{-\widetilde{G}_{n}(h-,, \cdot)}\left(h, \partial K_{n}(h)\right)$ has positive erosion of radius $\sigma h$.

As this lower bound of proximal radius does not depend on $n$ (but merely on $h, \lambda, \rho, K$ ), it is easy to prove indirectly for every $h \in] 0, \widehat{h}]$

So we obtain the estimate for every $h \in] 0, \widehat{h}]$,

$$
\mathbb{e}^{\supset}\left(\text { Graph }{ }^{b} N_{K}, \text { Graph }{ }^{b} N_{K_{n}}^{P} \mid P_{n, h}\right) \longrightarrow 0 \quad(n \longrightarrow \infty) .
$$

$$
\limsup _{n \longrightarrow \infty} \mathbb{e}^{\supset}\left(\operatorname{Graph}{ }^{b} N_{K}, \quad \text { Graph }{ }^{b} N_{K_{n}(h)}^{P}\right) \leq \operatorname{const}(\lambda) \cdot h .
$$

For proving transitional compactness of $\mathcal{K}_{\circ}\left(\mathbb{R}^{N}\right)$ in $\left(\mathcal{K}\left(\mathbb{R}^{N}\right), q_{\mathcal{K}, N}, \operatorname{LIP}_{\lambda}^{\left(\mathcal{H}_{\circ}^{\rho}\right)}\left(\mathbb{R}^{N}, \mathbb{R}^{N}\right)\right)$, a sequence $\left(h_{j}\right)_{j \in \mathbb{N}}$ in $\left.] 0, \widehat{h}\right]$ with $h_{j} \longrightarrow 0$ is given. Applying the Cantor diagonal construction, we obtain a subsequence (again denoted by) $\left(K_{n_{k}}\right)_{k \in \mathbb{N}}$ satisfying for every $j \in \mathbb{N}, k \geq j$

and thus, $\quad \limsup _{j \longrightarrow \infty} \sup _{k \geq j} q_{\mathcal{K}, N}\left(K, K_{n_{k}}\left(h_{j}\right)\right)=0$.

$$
\mathbb{e}^{\supset}\left(\text { Graph }{ }^{b} N_{K} \text {, Graph }{ }^{b} N_{K_{n_{k}}\left(h_{j}\right)}^{P}\right) \leq \operatorname{const}(\lambda) \cdot h_{j}+\frac{1}{k},
$$$$
\lim _{j \longrightarrow \infty} \sup _{k \geq j} q_{\mathcal{K}, N}\left(K, K_{n_{k}}\left(h_{j}\right)\right)=0
$$ 
Corollary 4.18 Let $f: \mathcal{K}\left(\mathbb{R}^{N}\right) \times[0, T] \longrightarrow \operatorname{LIP}_{\lambda}^{\left(\mathcal{H}_{\circ}^{\rho}\right)}\left(\mathbb{R}^{N}, \mathbb{R}^{N}\right)$ satisfy

$$
\left\|\mathcal{H}_{f\left(K_{1}, t_{1}\right)}-\mathcal{H}_{f\left(K_{2}, t_{2}\right)}\right\|_{C^{1}\left(\mathbb{R}^{N} \times \partial \mathbb{B}_{1}\right)} \leq \omega\left(q_{\mathcal{K}, N}\left(K_{1}, K_{2}\right)+t_{2}-t_{1}\right)
$$

for all $K_{1}, K_{2} \in \mathcal{K}\left(\mathbb{R}^{N}\right)$ and $0 \leq t_{1} \leq t_{2} \leq T$ with a modulus $\omega(\cdot)$ of continuity and consider the reachable sets of maps in $\operatorname{LIP}_{\lambda}^{\left(\mathcal{H}_{\circ}^{\rho}\right)}\left(\mathbb{R}^{N}, \mathbb{R}^{N}\right)$ as forward transitions on $\left(\mathcal{K}\left(\mathbb{R}^{N}\right), \mathcal{K}_{C^{1,1}}\left(\mathbb{R}^{N}\right), q_{\mathcal{K}, N}\right)$ according to Proposition 4.14.

Then for every initial set $K_{0} \in \mathcal{K}\left(\mathbb{R}^{N}\right)$, there exists a right-hand forward solution $K:[0, T[\longrightarrow$ $\mathcal{K}\left(\mathbb{R}^{N}\right)$ of the generalized mutational equation $\stackrel{\circ}{K}(\cdot) \ni f(K(\cdot), \cdot)$ with $K(0)=K_{0}$, i.e.

a) $\limsup _{h \downarrow 0} \frac{1}{h} \cdot\left(q_{\mathcal{K}, N}\left(\vartheta_{f(K(t), t)}(h, M), K(t+h)\right)-q_{\mathcal{K}, N}(M, K(t)) \cdot e^{10 \lambda h}\right) \leq 0$

for every compact $N$-dimensional submanifold $M \subset \mathbb{R}^{N}$ with $C^{1,1}$ boundary and $t \in[0, T[$.

b) $q_{\mathcal{K}, N}(K(s), K(t)) \leq \operatorname{const}(\lambda, T) \cdot(t-s) \quad$ for all $0 \leq s<t<T$.

Proof results from Proposition 4.17 along with Proposition 3.14 and Remark 3.15 (2.).

\section{A Reachable sets of differential inclusions in $\mathbb{R}^{N}$}

This appendix provides a collection of properties for the reachable sets of differential inclusions giving a quite general example of shape evolution. In particular, we use adjoint arcs for describing the timedependent limiting normal cones and find sufficient conditions for preserving smooth boundaries (for short times at least).

First we prove in Proposition A.2 that $C^{1,1}$ boundaries are preserved for short times even under slightly more general assumptions than $F \in \operatorname{LIP}_{\lambda}^{(\mathcal{H})}\left(\mathbb{R}^{N}, \mathbb{R}^{N}\right)$. Then according to Proposition A.4, the same hypothesis guarantees that the evolution of smooth sets is reversible in time. Finally, the conditions on the Hamiltonian function $\mathcal{H}_{F}$ are supposed to be stronger for guaranteeing that points evolve into sets of positive erosion. Details are presented in Proposition A.9.

\section{A.1 Hamiltonian system helps preserving $C^{1,1}$ boundaries shortly}

Definition A.1 For a set-valued map $F: \mathbb{R}^{N} \leadsto \mathbb{R}^{N}$, the standard hypothesis $(\mathcal{H})$ comprises the following conditions on $\mathcal{H}_{F}(x, p):=\sup p \cdot F(x)$

1. F has nonempty compact convex values,

2. $\mathcal{H}_{F}(\cdot, \cdot) \in C^{1,1}\left(\mathbb{R}^{N} \times\left(\mathbb{R}^{N} \backslash\{0\}\right)\right)$,

3. the derivative of $\mathcal{H}_{F}$ has linear growth, i.e. there is some $\gamma_{F}>0$ with $\left\|D \mathcal{H}_{F}(x, p)\right\|_{\mathcal{L}\left(\mathbb{R}^{N} \times \mathbb{R}^{N}, \mathbb{R}\right)} \leq \gamma_{F} \cdot(1+|x|+|p|) \quad$ for all $x, p \in \mathbb{R}^{N}(|p| \geq 1)$.

Proposition A.2 Assume standard hypothesis $(\mathcal{H})$ for $F: \mathbb{R}^{N} \leadsto \mathbb{R}^{N}$. For every initial set $K \in$ $\mathcal{K}_{C^{1,1}}\left(\mathbb{R}^{N}\right)$, there exist $\tau=\tau(F, K)>0$ and $\rho=\rho(F, K)>0$ such that $\vartheta_{F}(t, K)$ is also a $N-$ dimensional $C^{1,1}$ submanifold of $\mathbb{R}^{N}$ with boundary for all $t \in[0, \tau]$ and its radius of curvature is $\geq \rho \quad\left(\right.$ i.e. $\vartheta_{F}(t, K)$ has both positive reach and positive erosion of radius $\left.\rho\right)$.

The proof of Proposition A.2 is based on the following lemma : 
Lemma A.3 Suppose for $H:[0, T] \times \mathbb{R}^{N} \times \mathbb{R}^{N} \longrightarrow \mathbb{R}, \psi: \mathbb{R}^{N} \longrightarrow \mathbb{R}^{N}$ and the Hamiltonian system

the following properties :

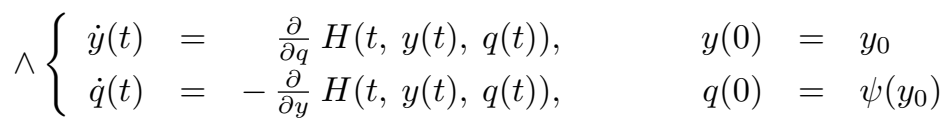

1. $H(t, \cdot, \cdot)$ is differentiable for every $t \in[0, T]$,

2. for every $R>0$, there exists $k_{R} \in L^{1}([0, T])$ such that the derivative of $H(t, \cdot, \cdot)$ is $k_{R}(t)$-Lipschitz continuous on $\mathbb{B}_{R} \times \mathbb{B}_{R}$ for almost every $t$,

3. $\psi$ is locally Lipschitz continuous,

4. every solution $(y(\cdot), q(\cdot))$ of the Hamiltonian system $(*)$ can be extended to $[0, T]$ and depends continuously on the initial data in the following sense:

Let each $\left(y_{n}(\cdot), q_{n}(\cdot)\right)$ be a solution satisfying $\quad y_{n}\left(t_{n}\right) \longrightarrow z_{0}, \quad q_{n}\left(t_{n}\right) \longrightarrow q_{0}$ for some $t_{n} \longrightarrow t_{0}, z_{0}, q_{0} \in \mathbb{R}^{N}$. Then $\left(y_{n}(\cdot), q_{n}(\cdot)\right)_{n \in \mathbb{N}}$ converges uniformly to a solution $(y(\cdot), q(\cdot))$ of the Hamiltonian system with $y\left(t_{0}\right)=z_{0}, \quad q\left(t_{0}\right)=q_{0}$.

For a compact set $K \subset \mathbb{R}^{N}$ and $t \in[0, T]$, define

$$
M_{t}^{\mapsto}(K):=\left\{(y(t), q(t)) \mid(y(\cdot), q(\cdot)) \text { solves system }(*), y_{0} \in K\right\} \subset \mathbb{R}^{N} \times \mathbb{R}^{N} .
$$

Then there exist $\delta>0$ and $\lambda>0$ such that $M_{t}^{\mapsto}(K)$ is the graph of a $\lambda$-Lipschitz continuous function for every $t \in[0, \delta]$.

Proof of Lemma A.3 follows exactly the same (indirect) track as [32, Frankowska 2002: Lemma 5.5] stating the corresponding result for the Hamiltonian system with $y(T)=y_{T}, q(T)=q_{T}$ given (without mentioning the uniform Lipschitz constant $\lambda$ explicitly).

Proof of Proposition A.2. $\quad$ Standard hypothesis $(\mathcal{H})$ for $F: \mathbb{R}^{N} \leadsto \mathbb{R}^{N}$ implies conditions (1.), (4.) of the preceding Lemma A.3 for the Hamiltonian $\mathcal{H}_{F}$.

Assuming that $K \in \mathcal{K}\left(\mathbb{R}^{N}\right)$ is a $N$-dimensional $C^{1,1}$ submanifold of $\mathbb{R}^{N}$ with boundary, the unit exterior normal vectors of $K$ (restricted to $\partial K$ ) can be extended to a Lipschitz continuous function $\psi: \mathbb{R}^{N} \longrightarrow \mathbb{R}^{N}$. Choosing some cut-off function $\varphi \in C^{\infty}\left(\left[0, \infty[,[0,1])\right.\right.$ with $\left.\varphi\right|_{\left[0, \frac{1}{4}\right]} \equiv 0,\left.\varphi\right|_{\left[\frac{1}{2}, \infty[\right.} \equiv 1$, $H(t, x, p):=\mathcal{H}_{F}(x, p) \cdot \varphi(|p|)$ satisfies condition (2.) of Lemma A.3 in addition.

For arbitrary $x_{0} \in \partial K$, consider now the differential equations

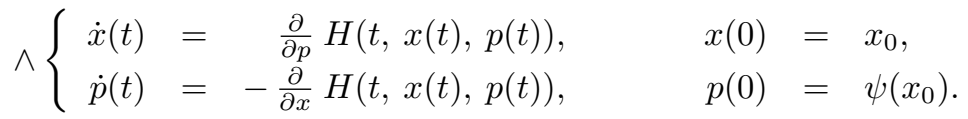

Due to $|\psi(\cdot)|=1$ on $\partial K$ and $H \in C^{1,1}$, there is $\tau_{1}>0$ such that $|p(t)|>\frac{1}{2}$ for all $t \in\left[0, \tau_{1}\right]$ and solutions $(x(\cdot), p(\cdot))$ of $(*)$ with $x_{0} \in \partial K$. Thus, $H=\mathcal{H}_{F}$ close to $(x(t), p(t))$. $\quad$ Now Proposition 4.5 can be reformulated as

$$
\text { Graph } N_{\vartheta_{F}(t, K)}(\cdot) \subset\left\{(x(t), \lambda p(t)) \mid(x(\cdot), p(\cdot)) \text { solves } \operatorname{system}(*), x_{0} \in \partial K, \quad \lambda \geq 0\right\},
$$

for all $t \in\left[0, \tau_{1}\right]$. Furthermore Lemma A.3 yields $\left.\tau \in\right] 0, \tau_{1}\left[\right.$ and $\lambda_{M}>0$ such that

$$
M_{t}^{\mapsto}(\partial K):=\left\{(x(t), p(t)) \mid(x(\cdot), p(\cdot)) \text { solves system }(*), x_{0} \in \partial K\right\}
$$

is the graph of a $\lambda_{M}$-Lipschitz continuous function for each $t \in[0, \tau]$.

Then for every point $z \in \partial \vartheta_{F}(t, K)$, the limiting normal cone $N_{\vartheta_{F}(t, K)}(z)$ contains exactly one direction and, its unit vector depends on $z$ in a Lipschitz continuous way. (The Lipschitz constant is uniformly bounded by $2 \lambda_{M}$ since the choice of $\tau_{1}$ ensures $|p(\cdot)|>\frac{1}{2}$ on $\left[0, \tau_{1}\right]$ for each solution of $(*)$.) So the compact set $\vartheta_{F}(t, K)$ is $N$-dimensional $C^{1,1}$ submanifold of $\mathbb{R}^{N}$ with boundary for all $t \in[0, \tau]$ and its radius of curvature has a uniform lower bound. 


\section{A.2 How to guarantee reversibility of $C^{1,1}$ sets in time (shortly)}

The Hamilton condition leads to a necessary condition on boundary points $x \in \partial \vartheta_{F}(t, K)$ and their limiting normal cones in Proposition 4.5. If each set $\vartheta_{F}(t, K) \quad(0 \leq t \leq T)$ has positive reach of radius $\rho$, then standard hypothesis $(\mathcal{H})$ turns adjoint arcs into sufficient conditions and, we conclude that the evolution of reachable sets is reversible with respect to time - in the sense of Proposition A.4.

Proposition A.4 Suppose standard hypothesis $(\mathcal{H})$ for the map $F: \mathbb{R}^{N} \leadsto \mathbb{R}^{N}$.

Assume for $K_{0} \in \mathcal{K}\left(\mathbb{R}^{N}\right)$ and $\rho>0$ that each compact set $K_{t}:=\vartheta_{F}\left(t, K_{0}\right) \quad(0 \leq t \leq T)$ has positive reach of radius $\rho$.

Then for every $0 \leq s \leq t<T, \quad K_{s}=\mathbb{R}^{N} \backslash \vartheta_{-F}\left(t-s, \mathbb{R}^{N} \backslash K_{t}\right)$.

Here we even suppose a uniform radius $\rho$ of positive reach for $K_{t} \stackrel{\text { Def. }}{=} \vartheta_{F}\left(t, K_{0}\right)$. The essential advantage for the proof is the relation between the boundaries of $K_{t} \subset \mathbb{R}^{N}$ and Graph $\left(t \longmapsto K_{t}\right) \subset \mathbb{R} \times \mathbb{R}^{N}$ stated in Proposition A.6 :

$$
\left.\partial \operatorname{Graph} \vartheta_{F}\left(\cdot, K_{0}\right)\right|_{[0, T]}=\left(\{0\} \times K_{0}\right) \cup \bigcup_{0<t<T}\left(\{t\} \times \partial \vartheta_{F}\left(t, K_{0}\right)\right) \cup\left(\{T\} \times \vartheta_{F}\left(T, K_{0}\right)\right) .
$$

Proof of Proposition A.4 $\vartheta_{F}\left(s, K_{0}\right) \subset \mathbb{R}^{N} \backslash \vartheta_{-F}\left(t-s, \mathbb{R}^{N} \backslash K_{t}\right)$ is an easy indirect consequence of definitions since it is equivalent to $\vartheta_{F}\left(s, K_{0}\right) \cap \vartheta_{-F}\left(t-s, \mathbb{R}^{N} \backslash K_{t}\right)=\emptyset$.

For proving the inverse inclusion indirectly at time $s=0$ (w.l.o.g.), we assume the existence of a time $t \in\left[0, T\left[\right.\right.$ and a point $y_{0} \in \mathbb{R}^{N}$ with $y_{0} \notin K_{0} \cup \vartheta_{-F}\left(t, \mathbb{R}^{N} \backslash K_{t}\right)$.

As an immediate consequence of $y_{0} \notin \vartheta_{-F}\left(t, \mathbb{R}^{N} \backslash K_{t}\right)$, the reachable set $\vartheta_{F}\left(t, y_{0}\right)$ is contained in $K_{t} \stackrel{\text { Def. }}{=} \vartheta_{F}\left(t, K_{0}\right)$. Now set $\tau:=\inf \left\{s \in[0, t] \mid \vartheta_{F}\left(s, y_{0}\right) \subset \vartheta_{F}\left(s, K_{0}\right)\right\}$.

In particular, $\quad \tau>0 \quad$ due to $y_{0} \notin K_{0}$. and $\quad \vartheta_{F}\left(\tau, y_{0}\right) \subset \vartheta_{F}\left(\tau, K_{0}\right) \quad$ due to the continuity of the reachable sets.

There are sequences $\tau_{n} \nearrow \tau$ and $\left(x_{n}(\cdot)\right)_{n \in \mathbb{N}}$ in $W^{1,1}\left([0, T], \mathbb{R}^{N}\right)$ satisfying

$$
\dot{x}_{n}(\cdot) \in F\left(x_{n}(\cdot)\right) \quad \text { a.e., } \quad x_{n}(0)=y_{0}, \quad x_{n}\left(\tau_{n}\right) \notin \vartheta_{F}\left(\tau_{n}, K_{0}\right) .
$$

Standard hypothesis $(\mathcal{H})$ and the compactness of trajectories (see e.g. [54, Theorem 2.5.3]) lead to subsequences (again denoted by) $\left(\tau_{n}\right)_{n \in \mathbb{N}}, \quad\left(x_{n}(\cdot)\right)_{n \in \mathbb{N}}$ and a solution $x(\cdot) \in W^{1,1}\left([0, T], \mathbb{R}^{N}\right)$ of $\dot{x}(\cdot) \in F(x(\cdot)) \quad$ (almost everywhere) with

$$
x_{n}(\cdot) \longrightarrow x(\cdot) \text { uniformly in }[0, T], \quad \dot{x}_{n}(\cdot) \longrightarrow \dot{x}(\cdot) \text { in } L^{1}\left([0, T], \mathbb{R}^{N}\right) .
$$

In particular, $(\tau, x(\tau))$ has to be a boundary point of Graph $\vartheta_{F}\left(\cdot, K_{0}\right)$. Proposition A.6 and $0<\tau \leq$ $t<T$ ensure $x_{\tau}:=x(\tau) \in \partial K_{\tau} \stackrel{\text { Def. }}{=} \partial \vartheta_{F}\left(\tau, K_{0}\right)$.

Moreover, $K_{\tau} \stackrel{\text { Def. }}{=} \vartheta_{F}\left(\tau, K_{0}\right)$ is supposed to have positive reach. So its limiting and proximal normal cone coincide at each boundary point and thus,

$$
\emptyset \neq N_{\vartheta_{F}\left(\tau, K_{0}\right)}\left(x_{\tau}\right)=N_{\vartheta_{F}\left(\tau, K_{0}\right)}^{P}\left(x_{\tau}\right) \subset N_{\vartheta_{F}\left(\tau, y_{0}\right)}^{P}\left(x_{\tau}\right) .
$$

For every unit vector $\nu \in N_{\vartheta_{F}\left(\tau, K_{0}\right)}\left(x_{\tau}\right)$, Proposition 4.5 leads to a solution $z(\cdot) \in C^{1}\left([0, \tau], \mathbb{R}^{N}\right)$ of $F$ and its adjoint arc $q(\cdot) \in C^{1}\left([0, \tau], \mathbb{R}^{N}\right)$ satisfying the corresponding Hamiltonian system and $z(0) \in K_{0}, \quad z(\tau)=x_{\tau}, q(\tau)=\nu$. Besides, the same Cauchy problem is solved by $x(\cdot)$ and its adjoint. $\mathcal{H}_{F} \in C^{1,1}$ implies the uniqueness of solutions and, its consequence $z(0)=x(0) \notin K_{0}$ leads to a contradiction.

Remark A.5 $\quad$ 1. The map $\mathcal{K}\left(\mathbb{R}^{N}\right) \leadsto \mathbb{R}^{N}, \quad K_{0} \longmapsto \mathbb{R}^{N} \backslash \vartheta_{-F}\left(t, \mathbb{R}^{N} \backslash \vartheta_{F}\left(t, K_{0}\right)\right)$ generalizes the morphological operation of closing (of sets in $\mathcal{K}\left(\mathbb{R}^{N}\right)$ ) that was introduced by Minkowski and is usually defined as $\mathcal{P}(X) \leadsto X, \quad K \longmapsto(K-t B) \ominus(-t B) \stackrel{\text { Def. }}{=}\{y \in X \mid y-t B \subset K-t B\}$ 
for a vector space $X$ and fixed $B \subset X, t>0$ (see e.g. [2, Aubin 99: Definition 3.3.1]).

2. In [9, Barron, Cannarsa, Jensen, Sinestrari 99], the viscosity solutions of the Hamilton-Jacobi equation $\partial_{t} u+H(t, x, D u)=0$ are investigated and roughly speaking, the continuous differentiability of $u$ is concluded from the reversibility in time :

If $u:[0, T] \times \mathbb{R}^{N} \longmapsto \mathbb{R}$ is a continuous viscosity solution of $\partial_{t} u+H(t, \quad \cdot, D u)=0$ and $\quad v(t, x):=u(T-t, x) \quad$ is a viscosity solution of $\partial_{t} v-H(T-t, \cdot, D v)=0$ then adequate assumptions of $H$ ensure $u \in C^{1}(] 0, T\left[\times \mathbb{R}^{N}\right)$.

Referring to the relation between reachable sets and level sets of viscosity solutions, we draw an inverse conclusion as we assume smoothness and obtain the reversibility in time.

3. The reversibility in time (in the sense of Proposition A.4) can also be regarded as recovering the initial data. Further results about this problem have already been published in [51, Rzeżuchowski 97] and [52, Rzeżuchowski 99], for example, but they usually assume other conditions. Either the initial set consists of only one point or the Hamiltonian function $\mathcal{H}_{F}$ is of class $C^{2}$.

Proposition A.6 Suppose for $F: \mathbb{R}^{N} \leadsto \mathbb{R}^{N}, K \in \mathcal{K}\left(\mathbb{R}^{N}\right)$ and $\rho>0$ that the map $[0, T] \leadsto \mathbb{R}^{N}$, $t \longmapsto \vartheta_{F}(t, K) \quad$ is $\lambda$-Lipschitz continuous (with respect to dl) and each set $\vartheta_{F}(t, K) \quad(0 \leq t \leq T)$ has positive reach of radius $\rho$.

Then the topological boundary of Graph $\left.\vartheta_{F}(\cdot, K)\right|_{[0, T]}$ in $\mathbb{R} \times \mathbb{R}^{N}$ is

$$
(\{0\} \times K) \cup \bigcup_{0<t<T}\left(\{t\} \times \partial \vartheta_{F}(t, K)\right) \cup\left(\{T\} \times \vartheta_{F}(T, K)\right) .
$$

Proof. The inclusion

$$
\left.(\{0\} \times K) \cup \bigcup_{0<t<T}\left(\{t\} \times \partial \vartheta_{F}(t, K)\right) \cup\left(\{T\} \times \vartheta_{F}(T, K)\right) \subset \partial \operatorname{Graph} \vartheta_{F}(\cdot, K)\right|_{[0, T]}
$$

is obvious. Due to the Lipschitz continuity of $\vartheta_{F}(\cdot, K)$, we only have to show

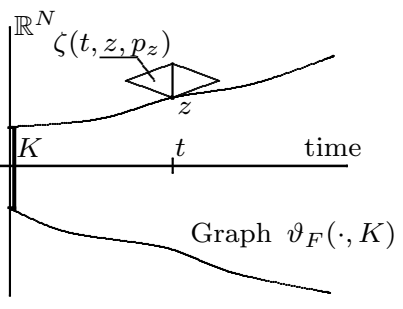

$$
\partial \operatorname{Graph} \vartheta_{F}(\cdot, K) \cap(] 0, T\left[\times \mathbb{R}^{N}\right) \subset \bigcup_{0<t<T}\{t\} \times \partial \vartheta_{F}(t, K) .
$$

Every point $z \in \partial \vartheta_{F}(t, K) \quad(0 \leq t \leq T)$ and any unit vector $p_{z} \in$ $N_{\vartheta_{F}(t, K)}^{P}(z)=N_{\vartheta_{F}(t, K)}(z)$ satisfy $\stackrel{\circ}{\mathbb{B}}_{\rho}\left(z+\rho p_{z}\right) \cap \vartheta_{F}(t, K)=\emptyset \quad$ and thus, $\quad\left(\{t\} \times \stackrel{\circ}{\mathbb{B}}_{\rho}\left(z+\rho p_{z}\right)\right) \cap \operatorname{Graph} \vartheta_{F}(\cdot, K)=\emptyset$.

The $\lambda$-Lipschitz continuity of $\vartheta_{F}(\cdot, K)$ implies

$$
\zeta\left(t, z, p_{z}\right) \cap \operatorname{Graph} \vartheta_{F}(\cdot, K)=\emptyset
$$

for $\zeta\left(t, z, p_{z}\right):=\left\{(s, y) \in \mathbb{R} \times \mathbb{R}^{N}|| z+\rho p_{z}-y|<\rho-\lambda| s-t \mid\right\}$.

Now choose $(t, x) \in \partial$ Graph $\vartheta_{F}(\cdot, K)$ with $0<t<T$ arbitrarily. The continuity of $\vartheta_{F}(\cdot, K)$ guarantees that Graph $\vartheta_{F}(\cdot, K)$ is closed and thus, it contains $(t, x)$.

Moreover there are sequences $\left(t_{n}\right)_{n \in \mathbb{N}},\left(x_{n}\right)_{n \in \mathbb{N}}$ in $] 0, T\left[, \mathbb{R}^{N}\right.$, respectively, satisfying $\left(t_{n}, x_{n}\right) \notin$ Graph $\vartheta_{F}(\cdot, K)$ for every $n \in \mathbb{N}$ and $\left(t_{n}, x_{n}\right) \longrightarrow(t, x)(n \longrightarrow \infty)$. For each $n \in \mathbb{N}$, let $z_{n}$ be an element of the projection $\Pi_{\vartheta_{F}\left(t_{n}, K\right)}\left(x_{n}\right) \subset \partial \vartheta_{F}\left(t_{n}, K\right)$.

Then, $\quad 0<\left|x_{n}-z_{n}\right|=\operatorname{dist}\left(x_{n}, \vartheta_{F}\left(t_{n}, K\right)\right) \leq\left|x_{n}-x\right|+\operatorname{dist}\left(x, \vartheta_{F}\left(t_{n}, K\right)\right) \longrightarrow 0$ and $\quad p_{n}:=\frac{x_{n}-z_{n}}{\left|x_{n}-z_{n}\right|} \in N_{\vartheta_{F}\left(t_{n}, K\right)}^{P}\left(z_{n}\right) \cap \partial \mathbb{B}_{1}$.

As mentioned before, we obtain $\quad \zeta\left(t_{n}, z_{n}, p_{n}\right) \cap \operatorname{Graph} \vartheta_{F}(\cdot, K)=\emptyset \quad$ for each $n \in \mathbb{N}$. Considering adequate subsequences (again denoted by) $\left(t_{n}\right)_{n \in \mathbb{N}},\left(x_{n}\right)_{n \in \mathbb{N}},\left(p_{n}\right)_{n \in \mathbb{N}}$ leads to the additional convergence $p_{n} \longrightarrow p \in \partial \mathbb{B}_{1}(n \longrightarrow \infty)$. So finally, $\zeta(t, x, p) \cap \operatorname{Graph} \vartheta_{F}(\cdot, K)=\emptyset$.

In particular, $\stackrel{\circ}{\mathbb{B}} \rho(x+\rho p) \cap \vartheta_{F}(t, K)=\emptyset$ implies $x \in \partial \vartheta_{F}(t, K)$. 


\section{A.3 How to make points evolve into sets of positive erosion}

Our aim consists in sufficient conditions for the positive erosion of $\vartheta_{F}(t, K)$. In [42, Lorenz 2005], sufficient conditions on $F$ have already been specified such that initial compact sets of positive erosion stay in $\mathcal{K}_{\circ}\left(\mathbb{R}^{N}\right)$. Weakening the assumption about the initial set (consisting now just of a single point), however, requires stronger properties of the set-valued map $F: \mathbb{R}^{N} \leadsto \mathbb{R}^{N}$ than standard hypothesis $(\mathcal{H})$ (see Definition A.1).

Definition A.7 For any $\rho>0$, a set-valued map $F: \mathbb{R}^{N} \leadsto \mathbb{R}^{N}$ satisfies the so-called standard hypothesis $\left(\mathcal{H}_{\circ}^{\rho}\right)$ if it has the following properties :

1. $F$ has convex values in $\mathcal{K}_{\circ}^{\rho}\left(\mathbb{R}^{N}\right)$,

2. $\mathcal{H}_{F}(\cdot, \cdot) \in C^{2}\left(\mathbb{R}^{N} \times\left(\mathbb{R}^{N} \backslash\{0\}\right)\right)$,

3. the derivative of $\mathcal{H}_{F}$ has linear growth, i.e. there is some $\gamma_{F}>0$ with

$$
\left\|D \mathcal{H}_{F}(x, p)\right\|_{\mathcal{L}\left(\mathbb{R}^{N} \times \mathbb{R}^{N}, \mathbb{R}\right)} \leq \gamma_{F} \cdot(1+|x|+|p|) \quad \text { for all } x, p \in \mathbb{R}^{N}(|p| \geq 1) .
$$

Remark A.8 Standard hypothesis $\left(\mathcal{H}_{\circ}^{\rho}\right)$ differs from its counterpart $(\mathcal{H})$ in two respects : The values of $F$ have uniform positive erosion (additionally) and its Hamiltonian is even twice continuously differentiable in $\mathbb{R}^{N} \times\left(\mathbb{R}^{N} \backslash\{0\}\right)$. This second restriction has the advantage that we can apply the tools of matrix Riccati equation (mentioned in Lemma A.11 and A.12).

\section{Proposition A.9}

Let $F_{1} \ldots F_{m}: \mathbb{R}^{N} \leadsto \mathbb{R}^{N}$ hold standard hypothesis $\left(\mathcal{H}_{\circ}^{\rho}\right)$ and

$$
\left\|\mathcal{H}_{F_{j}}\right\|_{C^{1,1}\left(\mathbb{R}^{N} \times \partial \mathbb{B}_{1}\right)} \stackrel{\text { Def. }}{=}\left\|\mathcal{H}_{F_{j}}\right\|_{C^{1}\left(\mathbb{R}^{N} \times \partial \mathbb{B}_{1}\right)}+\left.\operatorname{Lip} D \mathcal{H}_{F_{j}}\right|_{\mathbb{R}^{N} \times \partial \mathbb{B}_{1}}<\lambda
$$

for some $\lambda, \rho>0$. Moreover for a partition $0 \leq \tau_{0}<\tau_{1}<\ldots<\tau_{m}=1$ of $[0,1]$, define the map $\widetilde{G}:\left[0,1\left[\times \mathbb{R}^{N} \leadsto \mathbb{R}^{N}\right.\right.$ as $\widetilde{G}(t, x):=F_{j}(x)$ for $\tau_{j-1} \leq t<\tau_{j}$.

Furthermore choose $K \in \mathcal{K}\left(\mathbb{R}^{N}\right)$ arbitrarily.

Then there exist $\sigma>0$ and a time $\widehat{\tau} \in] 0,1]$ (depending only on $\lambda, \rho, K$ ) such that the reachable set $\vartheta_{\widetilde{G}}\left(t, x_{0}\right)$ has positive erosion of radius $\sigma t$ for any $\left.t \in\right] 0, \widehat{\tau}\left[, x_{0} \in K\right.$.

As an immediate consequence, $\vartheta_{\widetilde{G}}\left(t, K_{1}\right)$ has positive erosion of radius $\sigma t$ for all $\left.t \in\right] 0, \widehat{\tau}[$ and each initial subset $K_{1} \in \mathcal{K}\left(\mathbb{R}^{N}\right)$ of $K$.

The proof of this proposition uses matrix Riccati equations for Hamiltonian systems, but these tools of Lemma A.11 consider initial values induced by a Lipschitz function $\psi$. So roughly speaking, we exchange the two components $(x(\cdot), p(\cdot))$ (of a trajectory and its adjoint) preserving the Hamiltonian structure of their differential equations:

Lemma A.10 Assume the Hamiltonian system for $x(\cdot), p(\cdot) \in W^{1,1}\left([0, T], \mathbb{R}^{N}\right)$

$$
\dot{x}(t)=\frac{\partial}{\partial p} H_{1}(t, x(t), p(t)), \quad \dot{p}(t)=-\frac{\partial}{\partial x} H_{1}(t, x(t), p(t)) \quad \text { a.e. in }[0, T]
$$

with sufficiently smooth $H_{1}:[0, T] \times \mathbb{R}^{N} \times \mathbb{R}^{N} \longrightarrow \mathbb{R}$. Moreover set

$$
y(t):=-p(t), \quad q(t):=x(t) \quad H_{2}(t, \xi, \zeta):=H_{1}(t, \zeta,-\xi) .
$$

Then the absolutely continuous functions $(y(\cdot), q(\cdot))$ satisfy the Hamiltonian system

$$
\dot{y}(t)=\frac{\partial}{\partial q} H_{2}(t, y(t), q(t)), \quad \dot{q}(t)=-\frac{\partial}{\partial y} H_{2}(t, y(t), q(t)) \quad \text { a.e. in }[0, T] .
$$




\section{Lemma A.11}

In addition to the assumptions (2.)-(4.) of Lemma A.3, suppose for $\psi: \mathbb{R}^{N} \longrightarrow \mathbb{R}^{N}, H:[0, T] \times \mathbb{R}^{N} \times$ $\mathbb{R}^{N} \longrightarrow \mathbb{R}$ and the Hamiltonian system

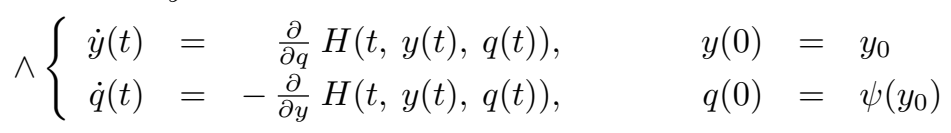

1'. $H(t, \cdot, \cdot)$ is twice continuously differentiable for every $t \in[0, T]$.

Then for every initial set $K \in \mathcal{K}\left(\mathbb{R}^{N}\right)$, the following statements are equivalent :

(i) For all $t \in[0, T], \quad M_{t}^{\mapsto}(K):=\left\{(y(t), q(t)) \mid(y(\cdot), q(\cdot))\right.$ solves $\left.(*), y_{0} \in K\right\}$ is the graph of a locally Lipschitz continuous function,

(ii) For any solution $(y(\cdot), q(\cdot)):[0, T] \longrightarrow \mathbb{R}^{N} \times \mathbb{R}^{N}$ of the initial value problem $(*)$ and each cluster point $Q_{0} \in \operatorname{Limsup}_{z \rightarrow y_{0}}\{\nabla \psi(z)\}$, the following matrix Riccati equation has a solution $Q(\cdot)$ on $[0, T]$

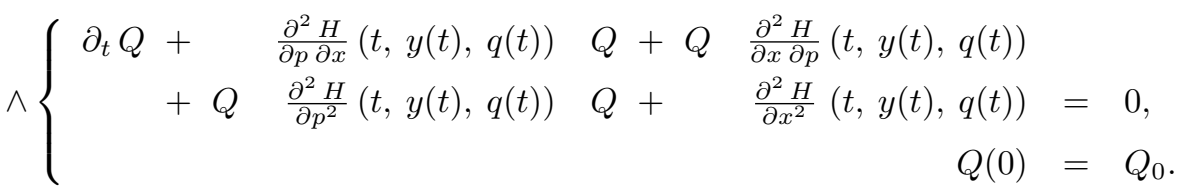

If one of these equivalent properties is satisfied and if $\psi$ is (continuously) differentiable, then $M_{t}^{\mapsto}(K)$ is even the graph of a (continuously) differentiable function.

Proof is given in [32, Frankowska 2002: Theorem 5.3], for the same Hamiltonian system but with $y(T)=y_{T}, q(T)=q_{T}$ given. So this lemma is an immediate consequence considering $-H(T-\cdot, \cdot, \cdot)$ and $(y(T-\cdot), q(T-\cdot))$.

For preventing singularities of $Q(\cdot)$, the following comparison principle provides a bridge to solutions of a scalar Riccati equation.

Lemma A.12 (Comparison theorem for the matrix Riccati equation, [50, Theorem 2]) Let $A_{j}, B_{j}, C_{j}:\left[0, T\left[\mathbb{R}^{N, N} \quad(j=0,1,2)\right.\right.$ be bounded continuous matrix-valued functions such that each $\quad M_{j}(t):=\left(\begin{array}{ll}A_{j}(t) & B_{j}(t) \\ B_{j}(t)^{T} & C_{j}(t)\end{array}\right) \quad$ is symmetric.

Assume that $U_{0}, U_{2}:\left[0, T\left[\longrightarrow \mathbb{R}^{N, N}\right.\right.$ are solutions of the matrix Riccati equation

$$
\frac{d}{d t} U_{j}=A_{j}+B_{j} U_{j}+U_{j} B_{j}^{T}+U_{j} C_{j} U_{j}
$$

with $M_{2}(\cdot) \geq M_{0}(\cdot) \quad\left(\right.$ i.e. $M_{2}(t)-M_{0}(t)$ is positive semi-definite for every $\left.t\right)$.

Then, given symmetric $U_{1}(0) \in \mathbb{R}^{N, N}$ with

$$
U_{2}(0) \geq U_{1}(0) \geq U_{0}(0), \quad M_{2}(\cdot) \geq M_{1}(\cdot) \geq M_{0}(\cdot),
$$

there exists a solution $U_{1}:\left[0, T\left[\longrightarrow \mathbb{R}^{N, N}\right.\right.$ of the corresponding Riccati equation with matrix $M_{1}(\cdot)$. Moreover, $\quad U_{2}(t) \geq U_{1}(t) \geq U_{0}(t) \quad$ for all $t \in[0, T[$.

Proof of Proposition A.9. The uniform bound $\lambda$ of $\left\|\mathcal{H}_{F_{j}}\right\|_{C^{1,1}\left(\mathbb{R}^{N} \times \partial \mathbb{B}_{1}\right)}(j=1 \ldots m)$ and Gronwall's Lemma lead to a radius $R=R(\lambda, K)>1$ and a time $T=T(\lambda, K) \in] 0,1[$

such that $1 . \vartheta_{\widetilde{G}}(t, K) \subset \mathbb{B}_{R}$ for all $t \in[0,1]$,

2. for every trajectory $x(\cdot)$ of $\widetilde{G}$ starting in $K$, each adjoint $p(\cdot)$ with

$$
\frac{1}{2} \leq|p(0)| \leq 2 \text { fulfills } \frac{1}{R}<|p(\cdot)|<R, \quad|p(\cdot)-p(0)|<\frac{1}{4 R} \quad \text { on }[0, T]
$$

So a smooth cut-off function again provides a map $H_{1}:[0, T] \times \mathbb{R}^{N} \times \mathbb{R}^{N} \longrightarrow \mathbb{R}$ that fulfills the assumptions of Lemma A.11 and is identical to $\mathcal{H}_{\widetilde{G}}$ in $[0, T] \times \mathbb{R}^{N} \times\left(\mathbb{R}^{N} \backslash \mathbb{B} \frac{1}{2 R}\right)$. 
Using the transformation of the preceding Lemma A.10, the auxiliary function

$$
H_{2}:[0, T] \times \mathbb{R}^{N} \times \mathbb{R}^{N} \longrightarrow \mathbb{R}, \quad(t, \xi, \zeta) \longmapsto H_{1}(t, \zeta,-\xi)
$$

is still holding the conditions of Lemma A.11. As a consequence, we obtain for any initial point $x_{0} \in K$ and time $\tau \in] 0, T]$ that the following statements are equivalent :

(i) For all $t \in[0, \tau]$, the set $M_{t}^{1}$ of all points $(p(t), x(t))$ with solutions

$(x(\cdot), p(\cdot)) \in W^{1,1}\left([0, t], \mathbb{R}^{N} \times \mathbb{R}^{N}\right)$ of

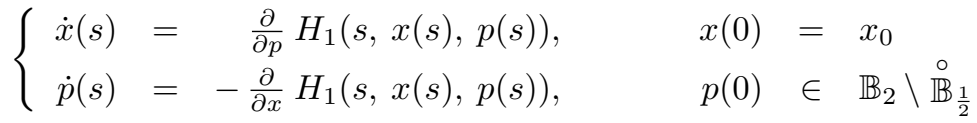

is the graph of a continuously differentiable function $f_{t}$.

(ii) For any solution $(x, p):[0, t] \longrightarrow \mathbb{R}^{N} \times \mathbb{R}^{N}$ of the initial value problem $(i)$ $(t \leq \tau)$, there exists a solution $Q:[0, t] \longrightarrow \mathbb{R}^{N \times N}$ of the Riccati equation

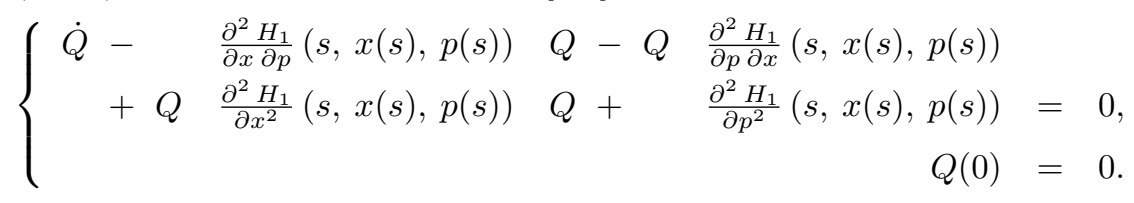

Now we give a criterion for the choice of $\widehat{\tau}$ : Setting

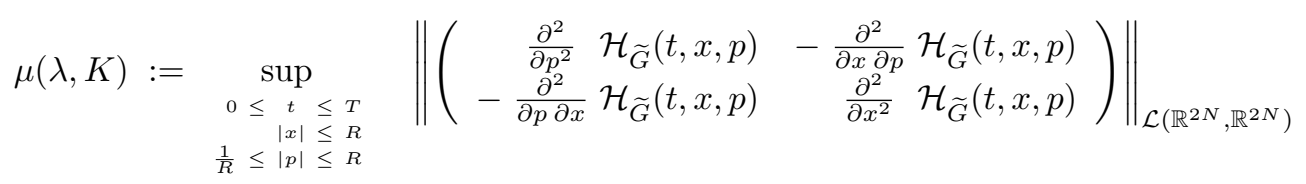

the comparison theorem for matrix Riccati equations (Lemma A.12) guarantees existence and uniqueness of such a solution $Q:[0, t] \longrightarrow \mathbb{R}^{N \times N}$ for any $t<\min \left\{T, \frac{\pi}{2 \mu}\right\}$ because for $a= \pm \mu$, the scalar Riccati equation $\frac{d}{d t} u=a+a u^{2}, u(0)=0$ has the solution $u(t)=\tan (a t)$ on $\left[0, \frac{\pi}{2|a|}[\right.$. Furthermore we obtain $\|Q(t)\| \leq \tan (\mu t)$.

Standard hypothesis $\left(\mathcal{H}_{\circ}^{\rho}\right)$ for $F_{1} \ldots F_{m}$ implies a constant $\sigma=\sigma(\lambda, \rho, K)>0$ with

$$
\xi \cdot \frac{\partial^{2}}{\partial p^{2}} \mathcal{H}_{\widetilde{G}}(t, x, p) \xi \geq 4 \sigma\left|\xi-\frac{\xi \cdot p}{|p|^{2}} p\right|^{2}
$$

for all $t \in[0, T],|x| \leq R, \frac{1}{R} \leq|p| \leq R, \xi$. Using the abbreviation

$$
D(t, x, p):=-\frac{\partial^{2} \mathcal{H}_{\widetilde{G}}}{\partial x \partial p}(t, x, p) Q(t)-Q(t) \frac{\partial^{2} \mathcal{H}_{\widetilde{G}}}{\partial p \partial x}(t, x, p)+Q(t) \frac{\partial^{2} \mathcal{H}_{\widetilde{G}}}{\partial x^{2}}(t, x, p) Q(t) \in \mathbb{R}^{N \times N},
$$
choose $\widehat{\tau}=\widehat{\tau}(\lambda, \rho, K)>0$ small enough such that

$$
\widehat{\tau}<\min \left\{T, \frac{\pi}{2 \mu}, \frac{1}{\lambda}\right\}, \quad\|D(t, x, p)\| \leq \sigma \quad \text { for every } t \in[0, \widehat{\tau}],|x| \leq R, \quad \frac{1}{R} \leq|p| \leq R .
$$

As a next step, we conclude that the solution $Q(t)$ of $(i i)$ (restricted to $[0, \widehat{\tau}]$ ) satisfies $Q(t) \leq$ $-\sigma t \cdot$ Id in the $(N-1)$-dimensional subspace of $\mathbb{R}^{N}$ perpendicular to $p(t)$. Indeed, let $(x(\cdot), p(\cdot)) \in$ $W^{1,1}\left([0, \widehat{\tau}], \mathbb{R}^{N} \times \mathbb{R}^{N}\right)$ be a solution of the Hamiltonian system $(i)$ and choose an arbitrary unit vector $\xi \in \mathbb{R}^{N}$ with $|\xi \cdot p(0)|<\frac{1}{4 R}$.

Then the auxiliary function $\quad \varphi:[0, \widehat{\tau}] \longrightarrow \mathbb{R}^{N}, t \longmapsto \xi \cdot Q(t) \xi+\sigma t\left|\xi-\frac{\xi \cdot p(t)}{|p(t)|^{2}} p(t)\right|^{2}$ satisfies $\varphi(0)=0$ and is absolutely continuous with $\dot{\varphi}(\cdot) \leq 0$ (due to $|p(t)-p(0)|<\frac{1}{4 R}$ and $\left.\frac{1}{R} \leq|p(t)| \leq R\right)$. So we obtain $\varphi(t) \leq 0$ for all $t \in[0, \widehat{\tau}]$.

Finally we need the geometric interpretation for concluding the positive erosion of $\vartheta_{\widetilde{G}}\left(t, x_{0}\right)($ of radius $\sigma t$ ) for each $t \in] 0, \widehat{\tau}\left[\right.$ and $x_{0} \in K$.

As mentioned before, the existence of the solution $Q(\cdot)$ on $[0, \widehat{\tau}[$ implies for all $t \in[0, \widehat{\tau}[$ that the set $M_{t}^{1}$ is graph of a $C^{1}$ function $f_{t}$. Moreover Proposition 4.5 guarantees 


$$
\text { Graph } N_{\vartheta_{\tilde{G}}\left(t, x_{0}\right)} \subset\{(x(t), \lambda p(t)) \mid(x(\cdot), p(\cdot)) \text { solves }(i), \lambda \geq 0\} \stackrel{\text { Def. }}{=} \bigcup_{\lambda \geq 0} \operatorname{Graph}\left(\lambda f_{t}^{-1}\right) .
$$

So we obtain for every $t \in] 0, \widehat{\tau}\left[\right.$ that each $p \in \mathbb{R}^{N} \backslash\{0\}$ belongs to the limiting normal cone of a unique boundary point $z \in \partial \vartheta_{\widetilde{G}}\left(t, x_{0}\right)$ and, $z=z(p)$ is continuously differentiable. In particular, the projection on $\vartheta_{\widetilde{G}}\left(t, x_{0}\right)$ is a single-valued function in $\mathbb{R}^{N}$ and thus, $\vartheta_{\widetilde{G}}\left(t, x_{0}\right)$ is convex for all $t \in] 0, \widehat{\tau}$ [ (see e.g. [21, Clarke,Stern,Wolenski 95: Corollary 4.12]). So it is sufficient to consider the limiting normal cones of $\vartheta_{\widetilde{G}}\left(t, x_{0}\right)$ locally at every boundary point.

Well-known properties of variational equations (see e.g. [32, Frankowska 2002]) and the uniqueness of solutions of the matrix Riccati equation $(i i)$ imply that $-Q(s)$ is the derivative of the $C^{1}$ function $f_{s}$ for $0<s \leq t<\widehat{\tau}$ (more details are presented in [43, Appendix A.7]). Thus for every time $t \in] 0, \widehat{\tau}[$, the derivative of $f_{t}$ at $p(t)$ is bounded by $\sigma t$ from below in a $(N-1)$-dimensional subspace of $\mathbb{R}^{N}$. Since $\vartheta_{\widetilde{G}}\left(t, x_{0}\right)$ is convex, it implies that $\vartheta_{\widetilde{G}}\left(t, x_{0}\right)$ has positive erosion of radius $\sigma t$.

Acknowledgments. The author would like to thank Prof. Willi Jäger for arousing the interest in set-valued maps and geometric evolution problems and furthermore, Prof. Jean-Pierre Aubin and Hélène Frankowska for their continuous support while working on this concept. He is also grateful to Irina Surovtsova and Daniel Andrej for fruitful complementary discussions.

Finally he acknowledges the financial support provided through the German Research Foundation, SFB 359 and the EC Human Potential Programme HPRN-CT-2002-00281.

\section{References}

[1] Ambrosio, L. (2000): Geometric evolution problems, distance function and viscosity solutions, in: Buttazzo, G. (ed.) et al., Calculus of variations and partial differential equations. Topics on geometrical evolution problems and degree theory, Springer-Verlag

[2] Aubin, J.-P. (1999): Mutational and Morphological Analysis: Tools for Shape Evolution and Morphogenesis, Birkhäuser-Verlag, Systems and Control

[3] Aubin, J.-P. (1993): Mutational equations in metric spaces, Set-Valued Analysis 1, pp.3-46

[4] Aubin, J.-P. (1992): A note on differential calculus in metric spaces and its applications to the evolution of tubes, Bull. Pol. Acad. Sci., Math. 40, No.2, pp.151-162

[5] Aubin, J.-P. (1991): Viability Theory, Birkhäuser-Verlag, Systems and Control

[6] Barles, G. \& Ley, O. (2006): Nonlocal first-order Hamilton-Jacobi equations modelling dislocations dynamics, Commun. Partial Differ. Equations 31, No.8, pp.1191-1208

[7] Barles, G., Soner, H.M. \& Souganidis, P.E. (1993): Front propagation and phase field theory, SIAM J. Control Optimization 31, No.2, pp.439-469

[8] Barles, G. \& Souganidis, P. (1998): A new approach to front propagation problems: theory and applications, Arch. Ration. Mech. Anal. 141, No.3, pp.237-296

[9] Barron, E.N., Cannarsa, P., Jensen, R. \& C. Sinestrari (1999): Regularity of Hamilton-Jacobi equations when forward is backward, Indiana Univ. Math. J. 48, No.2, pp.385-409

[10] Bellettini, G. \& Novaga, M. (1998): Comparison results between minimal barriers and viscosity solutions for geometric evolutions, Ann. Sc. Norm. Super. Pisa, Cl. Sci., IV. Ser. 26, No.1, pp.97-131

[11] Bellettini, G. \& Novaga, M. (1997): Minimal barriers for geometric evolutions, J. Differ. Equations 139, No.1, pp.76-103

[12] Brakke, K. (1978): The motion of a surface by its mean curvature, Princeton University Press

[13] Bressan, A. (1980): On two conjectures by Hájek, Funkcial. Ekvac. 23, pp.221-227.

[14] Cannarsa, P. \& Frankowska, H. (2006): Interior sphere property of attainable sets and time optimal control problems, ESAIM Control Optim. Calc. Var. 12, pp.350-370

[15] Cardaliaguet, P. (2001): Front propagation problems with nonlocal terms II, J. Math. Anal. Appl. 260, No.2, pp.572-601 
[16] Cardaliaguet, P. (2000): On front propagation problems with nonlocal terms, Adv. Differ. Equ. 5, No.1-3, pp.213-268

[17] Caroff, N. \& Frankowska, H. (1996): Conjugate points and shocks in nonlinear optimal control, Trans. Am. Math. Soc. 348, No.8, pp.3133-3153

[18] Chen, Yun-G., Giga, Y. \& Goto, S. (1991): Uniqueness and existence of viscosity solutions of generalized mean curvature flow equations, J. Differ. Geom. 33, No.3, pp.749-786

[19] Clarke, F.H. (1983): Optimization and Nonsmooth Analysis, Wiley-Interscience, Canadian Mathematical Society Series of Monographs and Advanced Texts

[20] Clarke, F.H., Ledyaev, Yu.S. \& Stern R.J. (1997): Complements, approximations, smoothings \& invariance properties, J. Convex Anal. 4, No.2, pp.189-219

[21] Clarke, F.H., Stern, R.J. \& Wolenski, P.R. (1995): Proximal smoothness and the lower- $C^{2}$ property, J. Convex Anal. 2, No.1/2, pp.117-144

[22] Cornet, B. \& Czarnecki, M.-O. (1999): Smooth normal approximations of epi-Lipschitzian subsets of $\mathbb{R}^{n}$, SIAM J. Control Optim. 37, No.3, pp.710-730

[23] Crandall, M., Ishii, H., Lions, P.-L. (1992): User's guide to viscosity solutions of second order partial differential equations, Bull. Am. Math. Soc., New Ser. 27, No.1, pp.1-67

[24] Crandall, M. \& Lions, P.-L. (1983): Viscosity solutions of Hamilton-Jacobi equations, Trans. Amer. Math. Soc. 277, No.1, pp.1-42

[25] Evans, L. \& Spruck, J. (1991): Motion of level sets by mean curvature. I, J. Differ. Geom. 33, No.3, pp.635-681

[26] Evans, L. \& Spruck, J. (1992): Motion of level sets by mean curvature. II, Trans. Amer. Math. Soc. 330, No.1, pp.321-332

[27] De Giorgi, E. (1994): Barriers, boundaries, motion of manifolds, Lectures held in Pavia, Italy

[28] Delfour, M.C., Zolésio, J.-P. (2006): Evolution equations for shapes and geometries, J. Evol. Equ. 6, pp.399-417

[29] Delfour, M.C., Zolésio, J.-P. (2001): Shapes and geometries. Analysis, differential calculus, and optimization, SIAM, Advances in Design and Control 4

[30] Federer, H. (1959): Curvature measures, Trans. Am. Math. Soc. 93, pp.418-491

[31] Federer, H. (1969): Geometric measure theory, Springer-Verlag, Grundlehren der mathematischen Wissenschaften 153

[32] Frankowska, H. (2002): Value function in optimal control, in: Agrachev, A. A. (ed.), Mathematical control theory, ICTP Lect. Notes. 8, pp.515-653

[33] Kelly, J.C. (1963): Bitopological spaces, Proc. Lond. Math. Soc., III. Ser. 13, pp.71-89

[34] Kloeden, P.E., Sadovsky, B.N. \& Vasilyeva, I. E. (2002): Quasi-flows and equations with nonlinear differentials. Nonlinear Anal. 51, No.7, pp.1143-1158

[35] Künzi, H.-P. (1992): Complete quasi-pseudo-metric spaces, Acta Math. Hung. 59, No.1/2, pp.121-146

[36] Kurzhanski, A.\& Vályi, I. (1997): Ellipsoidal calculus for estimation and control, Birkhäuser-Verlag, Systems and Control

[37] Kurzhanski, A.B. \& Varaiya, P. (2002): On reachability under uncertainty, SIAM J. Control Optimization 41, No.1, pp.181-216

[38] Kurzhanski, A.B. \& Nikonov, O.I. (1990): Funnel equations and multivalued integration problems for control synthesis, in: Perspectives in control theory, Proc. Conf., Sielpia/Pol. 1988, Prog. Syst. Control Theory 2, pp.143-153

[39] Lorenz, Th. (2007): Generalizing mutational equations for uniqueness of some nonlocal 1st-order geometric evolutions, to appear in Set-Valued Analysis, Preprint available at http://www .ub.uni-heidelberg.de/archiv/5580

[40] Lorenz, Th. (2007): Radon measures solving the Cauchy problem of the nonlinear transport equation, IWR Preprint available at http://www.ub.uni-heidelberg.de/archiv/7252

[41] Lorenz, Th. (2005): Evolution equations in ostensible metric spaces: Definitions and existence. IWR Preprint available at http://www.ub.uni-heidelberg.de/archiv/5519

[42] Lorenz, Th. (2005): Boundary regularity of reachable sets of control systems, Syst.Control Lett. 54, No.9, pp.919-924 
[43] Lorenz, Th. (2004): First-order geometric evolutions and semilinear evolution equations: A common mutational approach. Doctor thesis, Ruprecht-Karls-University of Heidelberg, available at http://www.ub.uni-heidelberg.de/archiv/4949

[44] Osher, S., Sethian, J. (1988): Fronts propagating with curvature-dependent speed: Algorithms based on Hamilton-Jacobi formulations, J. Comput. Phys. 79, No.1, pp.12-49

[45] Panasyuk, A.I. (1995): Quasidifferential equations in a complete metric space under conditions of the Carathéodory type. I, Differ. Equations 31, No.6, pp.901-910

[46] Panasyuk, A.I. (1992): Properties of solutions of a quasidifferential approximation equation and the equation of an integral funnel, Differ. Equations 28, No.9, pp.1259-1266

[47] Panasyuk, A.I (1985): Quasidifferential equations in metric spaces, Differ. Equations 21, pp.914-921

[48] Poliquin, R.A., Rockafellar, R.T \& Thibault, L. (2000): Local differentiability of distance functions, Trans. Am. Math. Soc. 352, No.11, pp.5231-5249

[49] Rockafellar, R.T. \& Wets, R. (1998): Variational Analysis, Springer-Verlag, Grundlehren der mathematischen Wissenschaften 317

[50] Royden, H.L. (1988): Comparison theorems for the matrix Riccati equation, Commun. Pure Appl. Math. 41, No.5, pp.739-746

[51] Rzeżuchowski, T. (1997): Boundary solutions of differential inclusions and recovering the initial data, Set-Valued Analysis 5, pp.181-193

[52] Rzeżuchowski, T. (1999): Continuous parameterization of attainable sets by solutions of differential inclusions, Set-Valued Analysis 7, pp.347-355

[53] Stoltenberg, R.A. (1969): On quasi-metric spaces, Duke Math. J. 36, pp.65-71

[54] Vinter, R. (2000): Optimal Control, Birkhäuser-Verlag, Systems and Control

[55] Wilson, W.A. (1931): On quasi-metric spaces, Amer. J. Math. 53, pp.675-684 